\title{
Quantitative energy-filtered electron microscopy of biological molecules in ice
}

\author{
John P. Langmore * and Michael F. Smith \\ Biophysics Research Division and Department of Biological Sciences, The Uniersity of Michigan, 2200 Bonisteel Boulevard, \\ Ann Arbor, MI 48109-2099, USA \\ Received at Editorial Office 9 April 1992
}

\begin{abstract}
The theoretical and experimental bases for quantitative electron microscopy of frozen-hydrated specimens are described, with special considerations of energy filtration to improve the images. The elastic and inelastic scattering from molecules in vacuum and in ice are calculated, and simple methods to approximate scattering are introduced. Multiple scattering calculations are used to describe the scattering from vitreous ice and to predict the characteristics of images of frozen-hydrated molecules as a function of ice thickness and accelerating voltage. Energy filtration is predicted to improve image contrast and signal-to-noise ratio. Experimental values for the inelastic scattering of ice, the energy spectrum of thick ice, and the contrast of biological specimens are determined. The principles of compensation for the contrast transfer function are presented. Tobacco mosaic virus is used to quantify the accuracy of interpreting image intensities to derive the absolute mass, mass per unit length, and internal mass densities of biological molecules. It is shown that compensation for the contrast transfer function is necessary and sufficient to convert the images into accurate representations of molecular density. At a resolution of $2 \mathrm{~nm}$, the radial density reconstructions of tobacoo mosaic virus are in quantitative agreement with the atomic model derived from $\mathrm{X}$-ray results.
\end{abstract}

\section{Introduction}

One of the primary goals of quantitative analysis of electron images is the determination of mass densities within biological molecules. In order to realize this goal the specimen structure must be well preserved and the image intensities must be quantitatively interpreted. The first requirement can be achieved by embedding the molecules in thin layers of vitreous ice. The second requirement might be difficult or impossible to achieve for frozen-hydrated molecules due to the uncertainties in correcting for the effects of phase contrast and inelastic scattering. This article shows that energy-filtered cryo-electron microscopy (cryo-EM) can accurately determine the absolute densities within biological molecules. The predictions of electron scattering and image

* To whom correspondence should be addressed. formation are reviewed and the advantages of energy filtration are discussed. Data are presented on inelastic scattering of vitreous ice and compared with predictions. Prediction and interpretation of the images of tobacco mosaic virus (TMV) are used to illustrate the ability to quantitatively interpret the images of frozen-hydrated biological molecules.

\subsection{Historical background}

Of the thousands of electron microscope (EM) studies of biological molecules there are very few that have tried to determine the internal densitics within biological molecules. At atomic resolution, the atom positions give unambiguous results, but there is only one study that has achieved such resolution [1]. A very common technique for determining density at lower resolution is to combine the phases derived from electron microscopy 
with amplitudes derived from electron or X-ray diffraction. However, this technique is usually limited to investigation of crystalline samples. Quantitative analysis of individual particles has been problematic, despite established procedures for averaging the information from many particles, due to inadequate information about the accuracy of the image amplitudes. The bulk of EM studies have focused on defining molecular outlines, using the techniques of shadowing, negative staining, etc. These studies are incapable of determining internal densities, mass per unit length, or total mass of biological molecules.

Using specialized techniques, bright-field and dark-field images of unstained dehydrated molecules have been used for quantitative microscopy (see, e.g., refs. [2-5]). The most accurate determinations of absolute mass come from dark-field scanning transmission electron microscopy (STEM) of unstained, frozen-dried biological specimens [6]. STEM analyses have the advantage of high image contrast without the use of phase contrast. The disadvantage of analyses of dehydrated molecules is the distortion caused by loss of water, which limits resolution to, at best, $2-4 \mathrm{~nm}$ [6]. The theory and practice of mass analysis of dried specimens has been adequately discussed in the reviews, above, and is outside the scope of this paper.

Methods to preserve and image biological molecules in thin layers of vitreous ice have created new possibilities for quantitative EM. Vitrification of biological specimens minimizes many of the artifacts associated with other preparative techniques [7-12]. Bright-field imaging of frozenhydrated specimens has emerged as a reliable, high-resolution technique to examine molecules in their native hydrated conformation. Cryoimages however, have low contrast because of the similarity between the density of biological material and the density of vitreous ice. The images also contain a high background of inelastic scattering. To overcome the low signal and high background, cryo-images are typically recorded 1-3 $\mu \mathrm{m}$ underfocus to maximize phase contrast. The introduction of phase contrast, however, significantly complicates quantitative analysis of the images.
A simplified theory of contrast formation described by Erickson and Klug [13] is usually used to understand the images. The relationship between object density and image contrast is described by the contrast transfer function ( $\mathrm{ClF}$ ), which combines the effects of interference between the unscattered and elastically scattered clectrons (phase contrast) and the effects from clcctron loss by scattering outside the objective aperture (scattering contrast). Under the defocused conditions that impart beneficial phase contrast to the images, the image intensities bear little resemblance to the object. Compensation (i.e., correction) for the CTF is theoretically possible, but implementation has been infrequent [13-15]. Most often the reciprocal space amplitudes have been compensated for phase contrast only, not compensated at all, or taken from electron or X-ray diffraction patterns. Some authors argue that scattering contrast is too weak to affect the images or that the CTF has little effect at low resolution. In fact, there is lack of confidence in compensation at low spatial frequencies, which are dominated by elastic and inelastic scattering contrast.

TMV is the ideal test specimen to establish whether the images of frozen-hydrated molecules can be used to accurately calculate molecular densities from the micrographs, since the atomic structure of the virus has becn determined [16]. Lepault [17] determined the radial density distribution of frozen-hydrated TMV without compensation of the CTF and without comparison of the results with $\mathrm{X}$-ray data. Jeng et al. [18] performed a three-dimensional image reconstruction of frozen-hydrated TMV and showed good qualitative agreement (at high resolution) with the electron density maps of Stubbs and coworkers [16]. However, they discarded the low-resolution electron amplitudes and instead used amplitudes calculated from the atomic model. They did not take into account inelastic scattering and did not consider the effects of solvent upon contrast. Although they could extract accurate phases to 1 $\mathrm{nm}$ resolution, they concluded that "the amplitudes determined by electron microscopy are in error, because of the difficulties in correcting accurately for the CTF". Therefore, these and 
other similar studies of frozen-hydrated molecules have not brought us to the important goal of determining the low-resolution structure of unknown molecules.

\subsection{Purpose of this paper}

This paper has the ultimate goal of showing how energy-filtered bright-ficld electron images of frozen-hydrated specimens can be used to determine the absolute mass densities of biological molecules. There are general discussions of elastic and inelastic scattering, including comparisons of the rigorous calculations with simple approximations that are useful for estimating contrast from molecules, and considerations of scattering from heavy atoms that might be useful as density labels. Because energy filtration has the potential for increasing the signal-to-noise ratio in the images and making the images more interpretable, therc are comparisons of image intensities with and without energy filtration. Image reconstruction is briefly reviewed, in the context of determination of the internal densities of molecules from the electron image intensities. Finally, the techniques of quantitative image reconstruction are illustrated using images of TMV in ice to calculate a radial density distribution that agrees quantitatively with X-ray results.

\section{Electron scattering}

Electrons are not absorbed by specimens embedded in layers of ice; they are scattered elastically or inelastically. Operating in different modes, the transmission EM can use these electrons to form images. Because the probability of electron scattering is greater for biological material than for ice, the number of scattered electrons increases in areas containing the molecules. By understanding the relationship between atomic composition and scattering probability, we can hope to understand the relationship between molecular density and image intensity.

Elastically scattcred electrons are scattered through large angles. The scattered electrons that are intercepted by the objective aperture are not transmitted into the image, forming scattering contrast (also called amplitude contrast). The elastic electrons that are transmitted by the objective aperture interfere with the unscattered electrons, forming phase contrast. Scattering contrast changes the total number of electrons in the image. Phase contrast moves the transmitted electrons from one place to another within the image. Control over phase contrast is achicved by defocusing the objective Iens, which changes the phase shift between the scattered and unscattered electrons. The elastic interaction is highly localized, allowing a high-resolution image to be formed.

Inelastically scattered electrons are scattered through very small angles and have undergone a loss of energy, which makes them subject to chromatic aberration. Electrons that are only inelastically scattered have a very high probability of transmission through the objective aperture. Electrons that have been seattered elastically as well as inclastically have the large angular distribution characteristic of elastic scattering and can be stopped by the aperture. In principle, an electron that is scattered inelastically and subsequently elastically could interfere with itself to form phase contrast, although this has never been demonstrated. In an electron microscope with a spectrometer, the inelastically scattered electrons can be efficiently removed by positioning the spectrometer slit to intercept the energy-loss clectrons [19]. The resolution of the inelastic scattering contrast is expected to be lower (perhaps 1-2 $\mathrm{nm}$ ) than that from the elastic scattering contrast, because of the increased range of the inelastic interactions $[20,21]$. The resolution limit of inelastic scattering contrast has not yet been fully characterized.

The first step in understanding the images is to predict the angular distribution of elastic scattering, and the energy and angular distribution of inclastic scattering. Those electrons transmitted by the objective aperture will contribute to the bright-field image.

\subsection{Calculation of atomic cross sections}

Electron scattering from molecules of known structure can be calculated by adding the scatter- 
ing due to the component atoms. The calculation of atomic scattering cross sections can be done using rigorous theory or by approximation using simple formulas. In the case of clastic scattering the rigorous theory is thought to give reliable results for higher angles, where the scattering is the same as for single atoms. At very small angles deviations from the single-atom predictions might occur due to chemical effects. Most calculations of elastic scattering in this paper are done from the single-atom partial-wave scattering amplitudes [22]. Simple approximations are introduced in order to allow the rapid estimation of electron scattering from biological molecules and heavy atoms. Inclastic scattering is more difficult to predict, due to the effects of chemical bonding upon the scattering probabilities. The predictions of inelastic scattering are largely untested by experiment. In this paper we will use a simple approximation to predict inelastic scattering from biological molecules.

\subsubsection{Elastic scattering}

2.1.1.1. Calculation of elastic cross sections from partial-wave scattering amplitudes Calculation of elastic scattering was based on the single-atom complex partial-wave solutions to elastic scattering from a Hartree-Fock potential [22]. The tables gave scattering intensitics that were comparable to those previously used [23]. The differen- tial phase shift of scattering was ignored, because the phase angles varied only slightly over the angles used for imaging. The magnitudes of atomic scattering were interpolated to 500 equally spaced angles using a four-point Lagrange method. The scattering amplitudes at different voltages were calculated by linear interpolation between the table amplitudes divided by $\gamma$, where $\gamma=1 /\left(1-\beta^{2}\right)$ and $\beta$ is the ratio of the electron velocity to that of light. Calculations at higher voltages were done by extrapolations from 100 $\mathrm{keV}$.

\subsubsection{Estimation of elastic scattering cross sec-} tions A simple formula for calculating the total elastic electron scattering cross section of an atom has been shown [23]. The recent values for the partial-wave scattering amplitudes [22] make it desirable to modify that equation slightly to the form:

$\sigma_{\mathrm{cl}}=\frac{1.4 \times 10^{-6} Z^{3 / 2}}{\beta^{2}}[1-0.26 Z /(137 \beta)] \mathrm{nm}^{2}$,

where $\sigma_{\mathrm{el}}$ is the total elastic scattering cross section $\left(\mathrm{nm}^{2}\right), Z$ the atomic number and $\beta$ the velocity of the electron divided by that of light $\left(\beta^{2}=1-\left[m c^{2} /\left(V_{0}+m c^{2}\right]^{2}\right)\right.$, where $V_{0}$ is the clectron acceleration voltage, and $m c^{2}$ the rest energy of the electron.

Table 1

Comparison of the partial-wave scattering cross sections at $100 \mathrm{keV}$ with predictions of eq. (4)

\begin{tabular}{|c|c|c|c|c|c|c|}
\hline $\begin{array}{l}\text { Atomic } \\
\text { number }\end{array}$ & $\begin{array}{l}\left.\sigma_{\mathrm{c}}(\mathrm{pm})^{2}\right) \\
(\mathrm{PW})\end{array}$ & $\begin{array}{l}\sigma_{\mathrm{e}}\left(\mathrm{pm}^{2}\right) \\
(\mathrm{eq} .(\mathrm{l}))\end{array}$ & $\begin{array}{l}\sigma_{\mathrm{c}} \eta_{\mathrm{c}}\left(\mathrm{pm}^{2}\right) \\
\left(s_{\mathrm{o}}=2 \mathrm{~nm}{ }^{1}\right) \\
(\mathrm{PW})\end{array}$ & $\begin{array}{l}\sigma_{\mathrm{c}} \eta_{\mathrm{c}}\left(\mathrm{pm}^{2}\right) \\
\left(s_{\mathrm{o}}=2 \mathrm{~nm}^{-1}\right) \\
(\text { eq. (4)) }\end{array}$ & $\begin{array}{l}\sigma_{\mathrm{c}} \eta_{\mathrm{c}}\left(\mathrm{pm}^{2}\right) \\
\left(s_{\mathrm{o}}=5 \mathrm{~nm}\right. \\
(\mathrm{PW})\end{array}$ & $\begin{array}{l}\sigma_{\mathrm{c}} \eta_{\mathrm{c}}\left(\mathrm{pm}^{2}\right) \\
\left(\mathrm{s}_{\mathrm{o}}=5 \mathrm{~nm}^{-1}\right) \\
(\mathrm{eq} .(4))\end{array}$ \\
\hline 1 & 3.65 & 4.65 & 3.01 & 3.72 & 1.53 & 2.33 \\
\hline 6 & 79.4 & 67.2 & 65.9 & 53.8 & 34.2 & 33.6 \\
\hline 7 & 85.1 & 84.4 & 79.6 & 67.5 & 43.9 & 39.8 \\
\hline 8 & 90.4 & 105 & 100 & 84.3 & 65.1 & 52.7 \\
\hline 11 & 148 & 164 & 116 & 131 & 73.5 & 82 \\
\hline 12 & 187 & 186 & 145 & 149 & 82.7 & 93 \\
\hline 15 & 314 & 257 & 255 & 206 & 133 & 129 \\
\hline 53 & $1730)$ & 1470 & 1460 & 1180 & 910 & 736 \\
\hline 74 & 2120 & 2210 & 1800 & 1790 & 1201 & 1110 \\
\hline 79 & 2110 & 2380 & 1880 & 1900 & 1300 & 1190 \\
\hline 80 & 2160 & 2420 & 1920 & 1940 & 1320 & 1210 \\
\hline
\end{tabular}


Table 2

Evaluation of the voltage dependence of eq. (1)

\begin{tabular}{|c|c|c|c|c|}
\hline \multirow{2}{*}{$\begin{array}{l}\text { Voltage } \\
(\mathrm{keV})\end{array}$} & \multicolumn{2}{|l|}{$Z=6$} & \multicolumn{2}{|l|}{$Z=80$} \\
\hline & $\begin{array}{l}\sigma_{\mathrm{e}}\left(\mathrm{pm}^{2}\right) \\
\text { (partial wave) }\end{array}$ & $\begin{array}{l}\sigma_{\mathrm{e}}\left(\mathrm{pm}^{2}\right) \\
(\mathrm{eq} .(1))\end{array}$ & $\begin{array}{l}\sigma_{\mathrm{e}}\left(\mathrm{pm}^{2}\right) \\
\text { (partial wave) }\end{array}$ & $\begin{array}{l}\sigma_{\mathrm{o}}\left(\mathrm{pm}^{2}\right) \\
(\mathrm{eq} \cdot(1))\end{array}$ \\
\hline 10 & 618 & 510 & 8550 & 5840 \\
\hline 40 & 170 & 142 & 3800 & 4250 \\
\hline 70 & 105 & 88.5 & 2710 & 3000 \\
\hline 100 & 79.4 & 67.2 & 2190 & 2420 \\
\hline
\end{tabular}

Table 1 is a comparison of the predictions of eq. (1) with the partial-wave calculations. Eq. (1) usually gives a value for the cross section that is within $15 \%$ of the partial-wave calculations based on the Hartree-Fock atomic potentials. The estimatc for hydrogen gives an exceptional error of almost $30 \%$. In eq. (1) the term in parentheses is a correction for failure of the first Born approximation, and is accurate in cases as long as $Z /(137 \beta) \leqslant 1.5$, as shown in table 2 . Thus for atoms with $Z<92$, the correction is accurate for electron voltages greater than $60 \mathrm{keV}$. This correction is insignificant for light atoms.

In the electron microscope, the elastic interactions cause electrons to be scattered through large angles. A large fraction of the elastically scattered electrons are stopped by the objective aperture, depending upon the angular acceptance of the aperture. It is common to relate the scattering angles to spatial frequencies using the equations:

$s=2 \sin (\alpha / 2) / \lambda$,

$s_{\mathrm{o}}=2 \sin \left(\alpha_{\mathrm{o}} / 2\right) / \lambda$,

where $\alpha$ is the scattering angle, $\alpha_{\mathrm{o}}$ the objective aperture half-angle, $s$ the spatial frequency, $s_{\mathrm{o}}$ the maximum spatial frequency and $\lambda$ the elcctron wavelength.

Commonly, objective apertures are used to stop electrons that have been scattered through angles greater than $s_{\mathrm{o}}=2-5 \mathrm{~nm}^{-1}$ (i.e., $\alpha_{\mathrm{o}}=$ $7.4-18.5 \mathrm{mrad}$ at $100 \mathrm{keV}$ ). The physical diameter of the aperture is $D=2 f \sin \alpha_{0}$, where $f$ is the focal length of the objective lens (usually 1-3 $\mathrm{mm}$ ). Removal of electrons by the aperture causes scattering contrast in a bright-field image. Thus, the number of electrons forming contrast is equal to the number scattered outside the aperture. The fraction of elastically scattered electrons that are scattered outside the objective aperture is called the elastic efficiency, $\eta_{\mathrm{e} 1}$. For an atomic number between 5 and 100 , and $s_{\mathrm{o}}=2-5 \mathrm{~nm}^{-1}$, the elastic cfficiency is in the range $0.4-0.9$. The effective clastic scattering atomic cross section is equal to the cross section, $\sigma_{\mathrm{el}}$, times $\eta_{\mathrm{el}}$. To a good approximation:

$$
\begin{aligned}
\sigma_{\mathrm{cl}} \eta_{\mathrm{cl}}= & \frac{1.4 \times 10^{-6} Z^{3 / 2}}{\beta^{2}}[1-0.26 Z /(137 \beta)] \\
& \times\left[1-s_{\mathrm{o}} / 10\right] \mathrm{nm}^{2},
\end{aligned}
$$

assuming that $s_{\circ}$ is in units of $\mathrm{nm}^{-1}$.

The second term in brackets is an approximation for $\eta_{\mathrm{el}}$ for single scattering, which is accurate to better than $20 \%$ over the range $1<s_{0}<5$ $\mathrm{nm}^{-1}$. To within an uncertainty of $<10 \%$, the partial wave valucs for $\eta_{\mathrm{el}}$ are linear in that range. Table 1 shows that eq. (4) is accurate to better than $20 \%$ for the atoms and voltages cxamined, except for hydrogen. For thick specimens multiple scattering will increase the value of $\eta_{\mathrm{el}}$ slightly.

\subsubsection{Inelastic scattering}

Biological molecules and ice scatter clectrons inelastically more frequently than elastically. For instance, at $100 \mathrm{keV}$ the ratio of inelastic-toelastic scattering probability for carbon is 1.6 [24]. The inelastic scattering is at very small angles such that in a conventional EM almost all of the electrons that have only been scattered inelastically are focused (with chromatic aberration) into the image and do not give rise to scattering contrast. In the absence of chromatic aberration, the effect of inelastic scattering would only be to decrease the coherence of the transmitted clectrons.

The use of a microscope with an electron spectrometer makes it possible to efficiently exclude the inelastically scattered electrons from the images and thus allow inelastic scattering to contribute to contrast. The design and operation of this type of microscope is discussed in refs. 
[25-29]. When all of the inclastically scattered electrons are removed from the image the inelastic scattering contributes efficiently to scattering contrast. As we will show later, the amount of inelastic scattering contrast from molecules in ice is predicted to be about four times greater than elastic scattering contrast. Thus it is imperative to have an accurate estimate of the inelastic scattering from specimens.

It is not possible to calculate the inelastic scattering cross sections with certainty, due to the effects of chemical bonding on the interactions with the outer-shell electrons. Wall ct al. [24] showed that the inelastic scattering cross sections for biological molecules can be approximated by the equation:

$\sigma_{\text {in }}=\frac{1.5 \times 10^{-6} Z^{1 / 2}}{\beta^{2}} \ln \left(2 / \vartheta_{\mathrm{c}}\right) \mathrm{nm}^{2}$,

where $\vartheta_{\mathrm{e}}=\bar{E} /\left[\beta^{2}\left(V_{\mathrm{o}}+m c^{2}\right)\right]$ and $\vec{E}$ is the average energy loss (assumed to be $20 \mathrm{eV}$ ). Wall et al. also derived an approximate equation for the differential scattering cross section, which we used to predict the angular distribution of the inelastic events (note that there is a typographical error in cq. (4) of Wall et al.; the exponent of $E_{\min }$ should be 2). As we will show later, in the Zciss EM902 the efficiency of filtering inelastic scattering from the images, $\eta_{\text {in }}$, is virtually unity. Because eq. (5) is not correct for hydrogen, we used an empirical hydrogen cross section of $8.8 \mathrm{pm}$, derived from the apparent mean free path for inelastic scattering in ice and the calculated inelastic scattering from oxygen.

\subsection{Scattering from biological molecules and heavy atoms}

The parameter that relates scattering probability to mass density is the mass scattering coefficient, $S_{\mathrm{m}}$, which is the scattering cross section per unit mass. In the case of the conventional EM it is appropriate to use the elastic scattering cross section $\left(\sigma \eta=\sigma_{\mathrm{cl}} \eta_{\mathrm{e})}\right)$, whereas in the encrgyfiltered EM it is necessary to use the sum of the elastic and inelastic cross sections $\left(\sigma \eta=\sigma_{\mathrm{e} 1} \eta_{\mathrm{e} 1}+\right.$ $\left.\sigma_{\text {in }} \eta_{\text {in }}\right)$. Thus, it is possible to predict the total scaltering from molecules of known molecular weight. More importantly, if we know the atomic composition of a sample, the empirical scattering cross sections can be used to determine the mass density, mass per unit length, or total mass. If we can assume a constant density for a molecule, the scattering probability can even be used to calculate thickness.

\subsubsection{Scattering in a vacuum}

In order to predict the effect of heavy atoms or biological molecules upon the images, we need to calculate the probability of scattering. The first step is to generalize the atomic cross sections to molecular cross sections and mass scattering coefficients. We can assume that the molecular cross section, $\sigma_{\mathrm{m}} \eta_{\mathrm{m}}$, is the sum of each of the cross sections, $\sigma(n)_{\eta}(n)$, of the atoms in the molecule:

$\sigma_{\mathrm{m} 1} \eta_{\mathrm{ml}} \approx \sum_{n=1}^{n=N_{\mathrm{m}}}[\sigma(n) \eta(n)]$,

which is the cross section of each molecule.

The mass scattering coefficient, $S_{m}$, is the molecular scattering cross section per unit mass:

$S_{\mathrm{m}}=\sigma_{\mathrm{m}} \eta_{\mathrm{m}} / M$

where $M$ is molccular weight (daltons).

Th probability of scattering from a uniform layer of material can be related to the bright-field images by the equation:

$I=I_{0} \operatorname{cxp}(-P)$,

where $I$ is the image intensity, $I_{0}$ the incident electron intensity and $P$ the probability of scattering in the layer:

$P=\sigma_{\mathrm{m}} \eta_{\mathrm{m}} \delta_{\mathrm{m}} t_{\mathrm{m}}$,

where $t_{\mathrm{m}}$ is the thickness of the molecular layer and $\delta_{\mathrm{m}}$ the number density of molecules.

Using the specific units of $\mathrm{g} / \mathrm{cm}^{3}$ for the mass density, $\rho_{\mathrm{m}}, \mathrm{nm}^{2}$ for the molecular scattering cross section, and nm for thickness, we obtain

$P=602 \sigma_{\mathrm{m}} \eta_{\mathrm{m}} \rho_{\mathrm{m}} t_{\mathrm{m}} / M$. 
The differences between the image intensity with and without the molecular layer, $\Delta I$, and the corresponding image contrast, $C$, are:

$$
\begin{aligned}
& \Delta I=I_{0}-I=I_{0}[1-\exp (-P)], \\
& C=\Delta I / I_{0} .
\end{aligned}
$$

For $P<0.1$ there is less than $5 \%$ error in the approximations:

$$
\begin{aligned}
& \Delta I \approx I_{0} P, \\
& C \approx P .
\end{aligned}
$$

A specimen will scatter a total number of electrons out of the image, $N$, given by:

$N=\int \Delta I(x, y) \mathrm{d} x \mathrm{~d} y$,

or

$N=\Delta I$ (area), for an area of uniform thickness.

In the thin-specimen approximation:

$N \approx \sigma \eta I_{0}$, for an atom,

$N \approx n \sigma \eta I_{0}$, for $n$ atoms,

$N \approx \sigma_{\mathrm{m}} \eta_{\mathrm{m}} I_{0}=S_{\mathrm{m}} M I_{0}$, for a molecule.

\subsubsection{Scattering from biological molecules in ice}

In order to calculate the contrast from molecules in ice, the excess scattering from the molecules must be determined. Excess scattering is the amount of scattering done by the molecules in excess of the scattering that would have occurred if only ice were present. The scattering done by a biological molecule is not much larger than that done by a comparable volume of water, because the atomic numbers of biological materials are similar to water. Thus the assumption about the amount of water displaced by the molecules is critical to the calculation of the molecular image. The uncertainties in these calculations are the density of ice and the partial specific volumes of the biological molecules in vitreous ice. Heavy atoms, on the other hand, do a large amount of scattering compared to that of the displaced water. Thus, heavy atom scattering is insensitive to ice embedment.

The partial specific volumes of biological molecules are measured in liquid aqueous solutions by determining the change in the volume of the solution upon addition of a known weight of dry biological material. The partial specific volume is thus a measurc of the molecular volume as well as any perturbation in the density of the water surrounding the molecules. At room temperature, the density of water bound to a macromolecule is slightly higher than that free in liquid solution [30]. At $100 \mathrm{~K}$ the density of bulk vitreous pure water is about $0.92 \mathrm{~g} / \mathrm{cm}^{3}$ [31], which is probably similar to that found in rapidly frozen thin layers [32]. However, the density of vitreous electrolyte solutions and the density of vitreous water bound to macromolecules are unknown.

The excess mass scattering coefficient of a molecule in water, $S_{\mathrm{m}}^{\prime}$, is:

$$
\begin{aligned}
S_{\mathrm{m}}^{\prime} & =S_{\mathrm{m}}-S_{\mathrm{s}} \rho_{\mathrm{s}} / \rho_{\mathrm{m}}=S_{\mathrm{m}}\left(1-S_{\mathrm{s}} \rho_{\mathrm{s}} / S_{\mathrm{m}} \rho_{\mathrm{m}}\right) \\
& =S_{\mathrm{rr}} F_{\mathrm{mms}},
\end{aligned}
$$

where $S_{\mathrm{s}}$ is the mass scattering coefficient for bulk solvent, $\rho_{\mathrm{s}}$ the density of bulk solvent, $\rho_{\mathrm{m}}$ the reciprocal of the partial specific volume and $F_{\mathrm{ms}}$ the resulting factor by which molecular scattering is multiplied due to displacement of solvent. It is a function of the molecular composition and solvent density.

Therefore, if we use units of $\mathrm{nm}^{2}$ for cross section, $\mathrm{nm}$ for thickness and $\mathrm{g} / \mathrm{cm}^{3}$ for density, the probability of excess scattering can be expressed as:

$P_{\mathrm{m}}^{\prime}=602 S_{\mathrm{m}}^{\prime} \rho_{\mathrm{m}} t_{\mathrm{m}}$.

The probability of scattcring from a frozen-hydrated specimen is the sum of the probability of scattering from the ice layer, $P_{\mathrm{s}}$, and the probability of excess scattering due to the molecule, $P_{\mathrm{m}}^{\prime}$. For an ice layer of thickness $t_{\mathrm{s}}$ and a molecule of thickness $t_{\mathrm{m}}$ :

$P_{\mathrm{s}+\mathrm{m}}=P_{\mathrm{s}}+P_{\mathrm{m}}^{\prime}=602\left(S_{\mathrm{s}} \rho_{\mathrm{s}} t_{\mathrm{s}}+S_{\mathrm{m}}^{\prime} \rho_{\mathrm{m}} t_{\mathrm{m}}\right)$,

$I_{\mathrm{s}}=I_{0} \exp \left(-P_{\mathrm{s}}\right)$,

$I_{\mathrm{s}+\mathrm{m}}=I_{0} \exp \left(-P_{\mathrm{s}+\mathrm{m}}\right)$,

$\Delta I=I_{\mathrm{s}}-I_{\mathrm{s}+\mathrm{m}}=I_{\mathrm{s}}\left[1-\exp \left(-P_{\mathrm{m}}^{\prime}\right)\right]$. 
The contrast of the molecule in ice is

$C=\Delta I / I_{\mathrm{s}}=\left[1-\exp \left(-P_{\mathrm{m}}^{\prime}\right)\right]$,

and for small $P_{\mathrm{m}}^{\prime}$,

$C \approx P_{\mathrm{m}}^{\prime}$.

\subsubsection{Elastic scattering from molecules in ice}

Calculated values of $\rho_{\mathrm{m}}, \eta_{\mathrm{m}}, S_{\mathrm{m}}, F_{\mathrm{m}}$ and $S_{\mathrm{m}}^{\prime}$ for elastic scattering from biological molecules and heavy atoms in vacuum and in ice are shown in table 3. The calculations assumed an incident voltage of $80 \mathrm{keV}$, an objective aperture of $s_{0}=$ $4.12 \mathrm{~nm}^{-1}$ and a density of $0.92 \mathrm{~g} / \mathrm{cm}^{3}$ for vitreous ice. We will show later that experimental measurements of the excess scattering from biological molecules can be used to test the predictions above.

The fact that the mass scattering coefficients for DNA, protein, and lipids in a vacuum are almost identical means that scattering from dry specimens is the same for different biological materials. This result is the basis for quantitative mass analysis by EM and STEM [2-6]. On the other hand, the excess elastic mass scattering coelficients of various molecules in ice are very different, because of the differences in the partial specific volumes. This leads to difficulties and opportunities for interpreting the images of molecules in ice. The difficulties are due to the sensitivity of the relative mass scattering cocfficients to the absolute density of ice. For instance the ratio of elastic scattering from DNA to that of protein is $1.85,2.15$ and 2.93 for ice densities of $0.82,1.0$ and $1.1 \mathrm{~g} / \mathrm{cm}^{3}$. Thus control of ice density is a significant hazard for quantitative microscopy in ice. On the other hand, the strong dependence upon solvent density leads to possibilities of performing contrast variation experiments in order to distinguish among nucleic acids, proteins, and lipids.

\subsubsection{Inelastic scattering from molecules in ice}

Table 3 also shows the predicted sum of elastic and inelastic excess mass scattering from molecules in ice. In this case, we have used the empirical value of the inelastic cross section from ice (see section 2.2.4) and the values of molecular scaltering from eq. (5). We assume that the spectrometer is able to filter the inelastically scattered electrons with an efficiency of 1 (see section 2.2.4). Although in vacuum the excess inclastic scattering is only slightly greater than clastic scattering, in vitreous ice the inelastic scattering is about 4 times greater than the elastic scattering. As a consequence, it should be expected that the bright-field EM contrast for hydrated molecules should be greatly improved by removal of the inelastically scattered electrons from the image.

Table 3 also shows that although the elastic scaltering from frozen-hydrated molecules is very dependent upon molecular composition, the sum of clastic and inelastic scattering is only slightly higher for nucleic acids than for protein. Thus, when the images are formed with just the zero-loss electrons, the expected mass scattering coefficients for different types of molecules are nearly identical. In other words, we expect that the

Table 3

Elastic and inelastic scattering from molecules in vacuum and vitrcous ice (80 $\left.\mathrm{keV}, s_{0}=4.12 \mathrm{~nm}{ }^{1}\right)$

\begin{tabular}{|c|c|c|c|c|c|c|c|c|c|}
\hline \multirow[t]{2}{*}{ Molecule } & \multirow{2}{*}{$\begin{array}{l}\rho_{\mathrm{m}} \\
\left(\mathrm{g} / \mathrm{cm}^{2}\right)\end{array}$} & \multicolumn{3}{|c|}{ Partial wave (PW) } & \multicolumn{3}{|c|}{ Elastic approximation (eq. (4)) } & \multicolumn{2}{|c|}{ PW elastic + inelastic } \\
\hline & & $\overline{\eta_{i}}$ & $\begin{array}{l}S_{\mathrm{m}} \\
\left(\mathrm{pm}^{2} / \mathrm{amu}\right)\end{array}$ & $\begin{array}{l}S_{\mathrm{m}} F_{\mathrm{ms}} \\
\left(\mathrm{pm}^{2} / \mathrm{amu}\right)\end{array}$ & $\overline{\eta_{\mathrm{e}}}$ & $\begin{array}{l}S_{\mathrm{m}} \\
\left(\mathrm{pm}^{2} / \mathrm{amu}\right)\end{array}$ & $\begin{array}{l}S_{\mathrm{m}} F_{\mathrm{mss}} \\
\left(\mathrm{pm}^{2} / \mathrm{amm}\right)\end{array}$ & $\begin{array}{l}S_{\mathrm{m}} \\
\left(\mathrm{pm}^{2} / \mathrm{amu}\right)\end{array}$ & $\begin{array}{l}S_{\mathrm{m}} F_{\mathrm{mls}} \\
\left(\mathrm{pm}^{2} / \mathrm{amu}\right)\end{array}$ \\
\hline lce & 0.92 & 0.658 & 4.24 & 0.0 & 0.6 & 4.47 & 0.0 & 14.27 & 0.0 \\
\hline Lipid & 1.03 & 0.539 & $4 .(1)$ & 0.21 & 0.6 & 4.03 & 0.037 & 15.19 & 2.47 \\
\hline Protein & 1.33 & 0.568 & 4.1 .3 & 1.19 & 0.6 & 4.14 & 1.05 & 15.23 & 5.35 \\
\hline DNA & 1.79 & 0.575 & 4.42 & 2.24 & 0.6 & 4.47 & 2.17 & 14.81 & 7.48 \\
\hline Iodine & 4.94 & 0.598 & 9.42 & 8.63 & 0.6 & 8.16 & 7.33 & 12.72 & 10.07 \\
\hline Gold & 19.3 & 0.680 & 8.33 & 8.13 & 0.6 & 8.39 & 8.18 & 10.93 & 10.25 \\
\hline
\end{tabular}


energy-filtered EM image intensities will be good representations of the mass densities for frozenhydrated molecules.

\subsubsection{Multiple scattering}

In some ways a thin molecule cmbedded in a thick layer of ice behaves as a "thin" object, because it causes a small increment in the image intensity. For instance, a TMV particle might have a contrast of $5 \%$ in a thick layer of ice, making eq. (27) valid. However, to determine the background intensity in the image of a thick layer of ice, multiple scattering must be taken into account. Multiple scattering changes the angular distribution of the scattered electrons and therefore affects the values of $\eta_{\mathrm{m}}$. In addition, as the sample becomes thicker, the ratio of energy-loss to no-loss clectrons in the image increases, which affects the ability of frozen-hydrated biological molecules to produce phase contrast. In order to gain any perspective on imaging molecules in ice, we must calculate the angular distribution of multiple scattering from layers of ice.

Crewe and Groves have described the considerations that must be made to calculate the image intensities for thick specimens and explicitly calculated the thickness contrast from carbonaceous specimens with and without energy filtration [33]. We have used a similar approach to calculate the density contrast from biological molccules $\mathrm{cm}$ bedded in thick layers of ice. We have also calculated the signal-to-noise ratios in the images. We have not taken into account the effects of beam broadening or chromatic aberration upon resolution, although those effects are predicted to dramatically reduce the resolution of the conventional bright-field EM $[34,35]$.

In order to calculate the angular distribution of the electrons that would be $n$-fold elastically scattered and $m$-fold inclastically scattered by a uniform layer of material, a delta function was convoluted $n$ times with the differential cross section for elastic scattering and convoluted $\mathrm{m}$ times with the differential cross section for inelastic scattering. The two-dimensional convolutions were performed for $80 \mathrm{keV}$, using table values for the elastic differential scattering cross sections [22] and the estimated values for the inelastic differential scattering cross sections [24]. An objective aperture of $s_{o}=4.12 \mathrm{~nm}^{-1}$ was assumed. For the ice thicknesses chosen in this study, all events beyond 9-fold scattering were ignored. Because the inelastic scattering was at very small angles, it would have becn fairly accurate to assume that the inelastic events gave no angular deviation in the path of the scattered electrons (the approximation used by Crewe and Groves [33]). However, for completeness, the predicted angular distribution of the inelastic scattering was taken into account. Disregard for scattering greater than 9 -fold contributed less than $0.1 \%$ error in the calculated scattering for an ice thickness of $500 \mathrm{~nm}$. In fact, calculated scattering beyond 5-lold contributed less than $8 \%$ of the total. The angular distributions of each type of multiple scattering (c.g., 3-fold elastic 2-fold inelastic) were calculated once and stored in the computer. In order to calculate the amount and angular distribution of scattering from an arbitrary thickness of ice, the multiple-scattering distributions were weighted by the Poisson statistical distribution, and added to give the predicted elastic and inelastic scattering distribution. This total scattering distribution was integrated from $s=0$ to $s_{0}$, in order to calculate the elastic and inelastic scattering within the aperture. Using the stored tables of multiple scattering distributions, calculation of the unfiltered and filtered image intensities for ice required less than $1 \mathrm{~ms}$ to compute on a Silicon Graphics 2500'T.

\subsubsection{Verification of the calculations and estima-} tion of the inelastic scattering cross section of ice

In principle, the scattering calculations could be checked by measuring the absolute intensities in the images of layers of ice of known thickness and density. Unfortunately, there are no studies of the physical thickness or density of the ice layers used for cryo-EM. However, the relative amounts of scattering outside the objective apcrture and energy-loss scattering inside the aperture can be measured using a microscope with an energy filter, such as the Zeiss EM902, and compared with the calculations.

We collected more than 20 sets of low-dose micrographs of different areas on a frozen-hy- 
drated specimen $\left(V_{0}=80 \mathrm{keV}\right.$, dose $=600$ electrons $/ \mathrm{nm}^{2}$, incident dose rate $=200$ electrons $\mathrm{nm}{ }^{2} \mathrm{~s}^{-1}, s_{0}=4.12 \mathrm{~nm}^{-1}$, and $T=116 \mathrm{~K}$ ). The methods of microscopy and densitometry are described elsewhere [36,37]. Briefly, frozen-hydrated specimens over holey carbon films were scanned to find areas of different apparent ice thickness. The microscope was focused at 80000 $x$ magnification on the carbon using off-axis convergent illumination. The images were recorded by electronically spreading the illumination and shifting the heam on axis for the desired exposure time using a modified microdose focusing system on the Zeiss EM902. Two micrographs were recorded at $30300 \times$ magnification on each area of ice, the first with a spectrometer slit (17 $\mathrm{eV}$ energy window) adjusted to include only the no-loss elcctrons transmitted by the aperture, and the second with the spectrometer slit removed to record all transmitted electrons. The incident illumination was recorded twice by imaging an empty hole before and after imaging the ice. The illumination was confirmed to be constant. The film (Kodak SO 163) was developed for $12 \mathrm{~min}$ in full strength $\mathrm{D} 19$ at $68^{\circ} \mathrm{C}$. The electron image densities were measured with a CCD camera interfaced to a computer. Film density was calculated from transmitted light using the equation

$D(x, y)=-\log \left[T(x, y) / T_{0}(x, y)\right]$,

where $T(x, y)$ was the intensity of light transmitted by an image of ice and $T_{0}(x, y)$ was the intensity transmitted by the light box without the micrograph. The absolute accuracy of the densitometry was confirmed for the density range $0-2$. The effects of optical flare in the CCD camera lens were minimized by masking off areas of fairly uniform density. The electron intensity was calculated using the relationship $I(x, y)=$ $K\left[D(x, y)-D_{0}\right]$, where $D_{0}$ was the density of the film without any electron exposure and $K$ was the film speed. The value of $K$ was about 0.45 electrons $/ \mu \mathrm{m}^{2}$, but was not important for later analysis. Magnification was calculated to be $30300 \times$, from the position of the $2.3 \mathrm{~nm}$ third layer line of TMV. Image processing was performed using the EMPRO program package [36] on a Silicon Graphics IRIS 2500T workstation

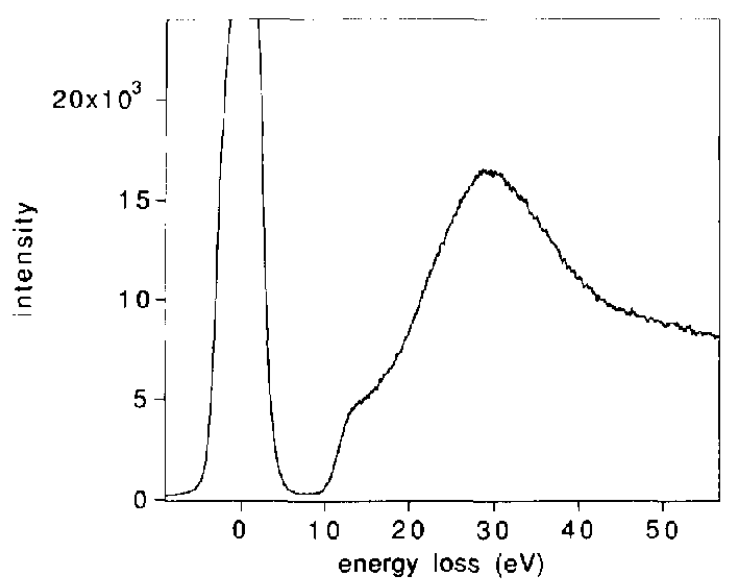

Fig. 1. Energy-loss spectrum of electrons transmitted by a layer of vitreous ice about $140 \mathrm{~nm}$ thick through an objective aperture of $s_{0}=4.12 \mathrm{~nm}^{-1}$. For recording energy-filtered images, the spectrometer slit transmits electrons of energy loss $0.10 \mathrm{eV}$ at the center of the image area and $0-2 \mathrm{eV}$ at the edge of the image area. Even at the center of the images more than $99 \%$ of the energy-loss electrons are intercepted.

(Silicon Graphics, Inc., Mountain Vicw, CA).

In order to compare the scattering predictions to the experimental image intensities, we first need to know the fraction of the inelastically scattered electrons that are removed by the spectrometer. Fig. 1 shows the electron energy spectrum found for a layer of ice about $140 \mathrm{~nm}$ thick. The efficiency of removal of energy-loss electrons by the $17 \mathrm{eV}$ slit was greater than 0.99 , and subsequently assumed to be 1 .

In order to cstimate the thickness of the ice, we relicd upon the theoretical clastic scattering cross sections, assuming an ice density of 0.92 $\mathrm{g} / \mathrm{cm}^{3}$. The ice thickness was calculated from the ratio of the unfiltered image intensity to the incident intensity, assuming a $280 \mathrm{~nm}$ mean frec path for elastic scattering.

Using the procedures outlined in section 2.1.2, the intensitics of the total transmitted electrons and of the zero-loss transmitted electrons were predicted using the theoretical Hartree-Fock values for the elastic cross section of ice and different trial values for the inclastic cross section. These predictions were compared to the empirical unfiltered and energy-filtered intensities for image pairs recorded from different thicknesses of ice. The calculations fit the image data if the 
empirical inelastic cross section of ice was assumed to be $181 \mathrm{pm}^{2}$. Assuming an ice density of $0.92 \mathrm{~g} / \mathrm{cm}^{3}$, the theoretical mean frec path for elastic scattering outside the $90 \mu \mathrm{m}$ aperture was $280 \mathrm{~nm}$, and the empirical mean free path for inelastic scattering was $180 \mathrm{~nm}$. The comparison of the predictions and experimental data is shown in fig. 2. The predictions were very sensitive to the value chosen for the inelastic mean free path. The ratio of unfiltered to filtered ice image intensity $\left(I_{- \text {spec }} / I_{+ \text {spec }}\right)$ is 1 for very thin ice, but increases to about 2.8 for $200 \mathrm{~nm}$ of ice and to over 12 for $450 \mathrm{~nm}$ of ice, as inelastic scattering accumulates in the image. Thus for $200 \mathrm{~nm}$ and $450 \mathrm{~nm}$ of ice over $50 \%$ and $90 \%$, respectively, of the electrons in the image have becn inelastically scattered. The good agreement between the predictions and the experimental data indicate that our method of calculating the images of ice is valid up to at least $450 \mathrm{~nm}$ thickness.

\section{Role of energy filtration in imaging frozen-hy- drated molecules}

In order to compare the energy-filtered and unfiltered images, we calculated the images of thick layers of ice and estimated the incremental

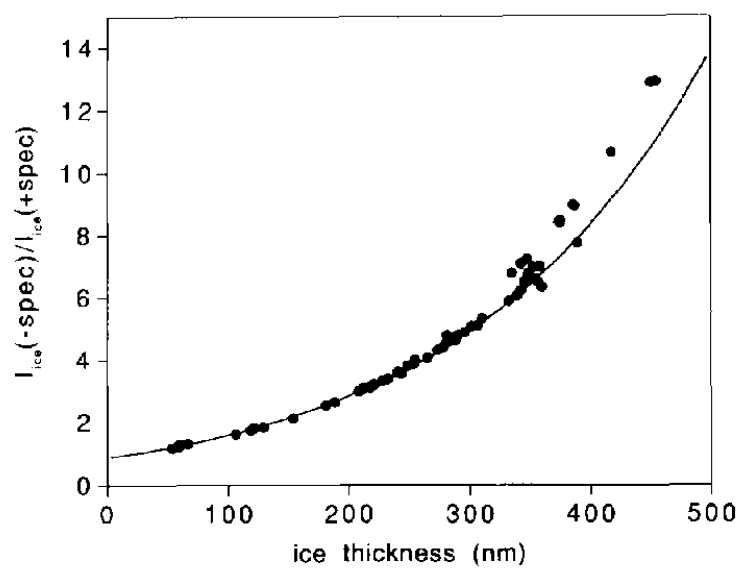

Fig. 2. Ratio of ice image intensities without and with encrgy filtration. This ratio is very sensitive to the relative mean free paths for elastic and inelastic scattering. (—) Predictions;

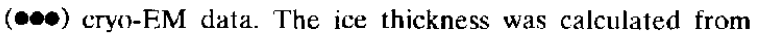
the theoretical mean free path for elastic scattering at $80 \mathrm{keV}$, $s_{0}=4.12 \mathrm{~nm}^{-1}$.

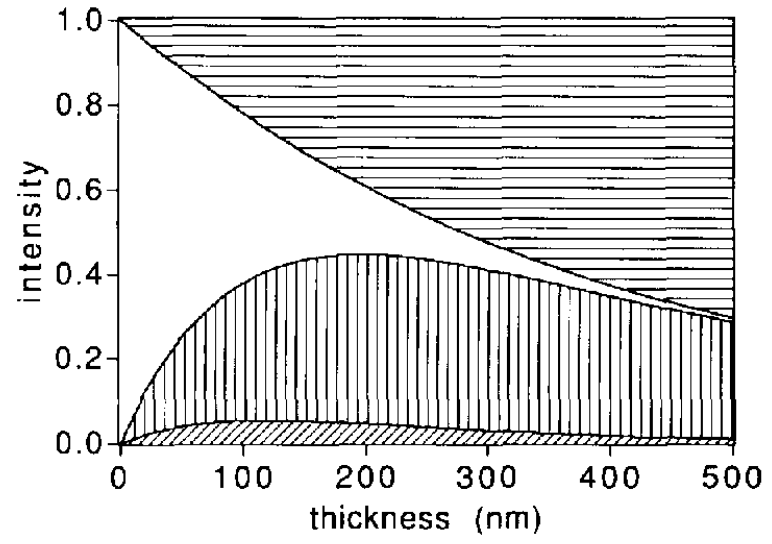

Fig. 3. Intensities of the four categories of electrons relevant to image formation in conventional and energy-filtered EM at $80 \mathrm{keV}$ and $s_{1}=4.12 \mathrm{~nm}^{-1}$, as a function of apparent ice thickness: (diagonally hatched area) elastically scattered electrons within the aperture, $I_{c}$; (vertically hatched area) energy-loss within the aperture, $I_{\text {incl }}$; (clear area) unscattered electrons, $I_{\text {un }}$ ( (horizontally hatched area) electrons scattered outside the objective aperture, which do not contribute to the image.

scattering caused by a molccule embedded within the ice. Using the algorithms and results in section 2, we predicted the angular distribution of the intensity of the no-loss and energy-loss electrons for different thicknesses of ice. Fig. 3 is a summary of the intensities of the three categories of electrons relevant to image formation (unscattered, $I_{\text {un }}$; inelastically scattered within the aperture, $i_{\text {inel }}$; and elastically scattered within the aperture, $I_{\mathrm{c}}$ ) at $80 \mathrm{keV}$ and $s_{\mathrm{o}}=4.12 \mathrm{~nm}^{-1}$ as a function of ice thickness. It is clear that the inelastically scattered electrons dominate the imagcs of ice greater than $\sim 150 \mathrm{~nm}$ thick. Removal of the inelastically scattered electrons has the potential to dramatically affect the images. In this section we will illustrate the effects of cnergy filtration upon ice background, specimen contrast, and signal-to-noise ratio. Fnergy-filtered imaging at $80 \mathrm{keV}$ will be compared to that at higher voltages.

\subsection{Calculations of ice background and virus con- trast with and without energy filtration}

The ice image intensity in the unfiltered microscope is $I_{\text {-spec }}=I_{\text {un }}+I_{\text {inel }}+I_{\mathrm{e}}$, and that in the 
encrgy-filtered microscope is $I_{\text {spec }}=I_{\mathrm{un}}+I_{\mathrm{c}}$. Fig. 4 shows calculated values for $I$ spec and $I_{1 \text { spec }}$ as a function of ice thickness at $80 \mathrm{keV}$ and $s_{0}=4.12 \mathrm{~nm}^{-1}$. These two quantities are predictions of the background intensities in the images of molecules embedded in ice. The increments in elastic and inelastic scattering due to the presence of a TMV particle (see section 4.2) were also calculated. Fig. 5 compares the calculated peak TMV scattering signals, $\Delta I$, and the ratio of scattering contrast with and without energy filtration.

For very thin layers of ice, the background intensity does not depend upon encrgy filtration, because most of the elcctrons in the image are unscattered. In thin ice the scattering signal is 4 times greater in the filtered than in the unfiltered images, because of the additional, inclastic contrast. The signal advantage is simply the ratio of the total excess scattering cross section to the elastic excess scattering cross section (see table $3)$. The net result is that the scattering contrast is 4 times greater when energy filtration is used.

For moderate ice thickness (e.g., $150 \mathrm{~nm}$ ), energy filtration reduces the ice background by a factor of 2.2, and increases the scattering signal by a factor of 1.7 . In the case of the conventional EM, clectrons that have been scattered inclastically are still available for elastic scattering from

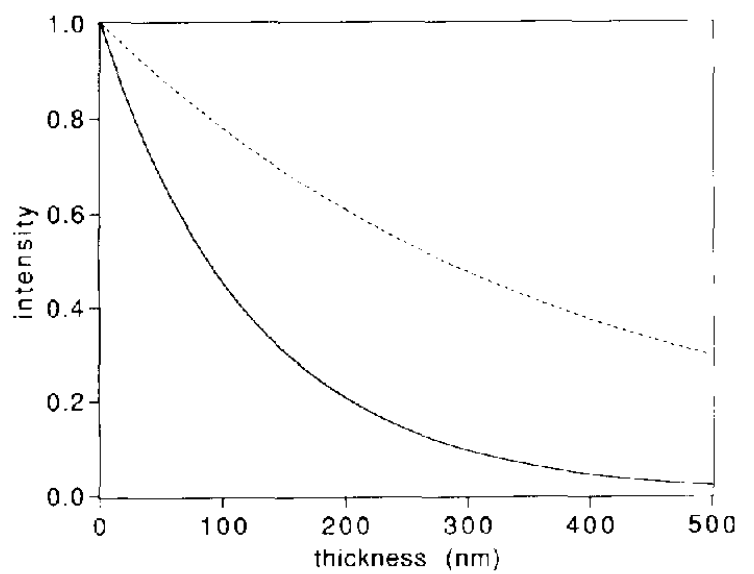

Fig. 4. Calculated values for ice background as a function of ice thickness at $80 \mathrm{keV}$ and $s_{0}=4.12 \mathrm{~mm}^{-1}:(-)$ with energy filtration; (.....-) without filtration.

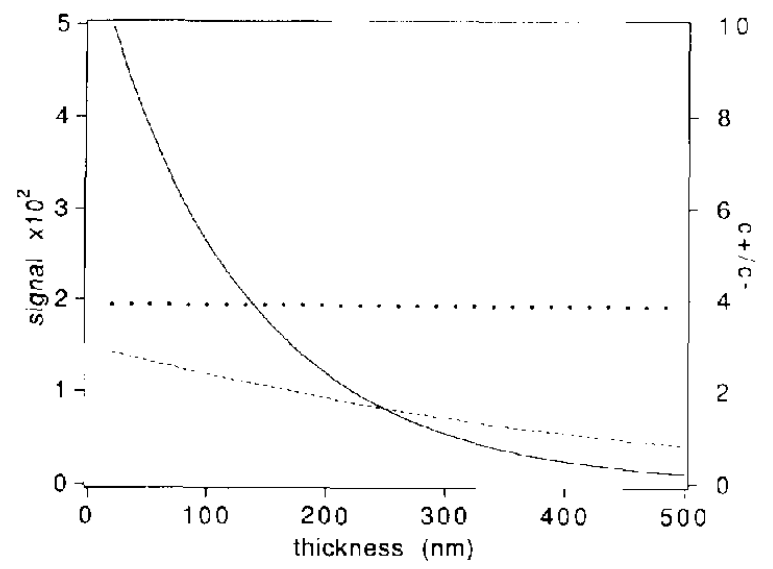

Fig. 5. Calculated peak scaltering signals and contrast for TMV in vitreous ice at $80 \mathrm{keV}$ and $s_{0}=4.12 \mathrm{~nm} \mathrm{I}^{\prime}:(\longrightarrow$ $\Delta I$ with energy filtration; (.....) $\Delta I$ without filtration; $(\ldots \ldots)$ ratio of TMV contrast with and without energy filtration. Contrast defined by eq. (26). Method of modeling TMV from ref. [37].

the TMV. In the case of the energy-filtered FM, the available electrons are becoming scarce, because most of them have been removed by the spectrometer and aperture. The net result is that the contrast is 3.8 times greater when energy filtration is used.

For very thick ice (c.g., $500 \mathrm{~nm}$ ) the ice background is 15 times less in the filtered images, but the scattering signal is 3.4 times less, due to the paucity of transmitted electrons. The net result is that the contrast is 4 times greater when energy filtration is used. The striking result is that the contrast advantage resulting from energy filtralion is predicted to be $\sim 4$, regardless of ico thickness.

3.2. Calculations of signal-to-noise ratios in the images of a cirus with and without energy filtration

The most important parameter in image formation is not contrast, but signal-to-noise ratio $(\mathrm{S} / \mathrm{N})$. The depletion of electrons by energy filtration not only causes the ice background to decrease (a beneficial reduction in statistical noise), but decreases the scattering signal (a detrimental reduction in signal). The $\mathrm{S} / \mathrm{N}$ can be calculated from the increment in the image inten- 
sity caused by the presence of a molecule divided by the standard deviation in the total number of electrons in an equal area of the image. For purposes of understanding our studies of TMV at $2 \mathrm{~nm}$ resolution [37,38] it is reasonable to choose a molecular area of $1 \mathrm{~nm}^{2}$, and an incident intensity of 600 electrons per $\mathrm{nm}^{2}$ at $80 \mathrm{keV}$ and $s_{\mathrm{o}}=4.12 \mathrm{~nm}^{-1}$. Obviously the same incident electron dose must be chosen for both the energyfiltered and unfiltered cases, so that the resultant radiation damage would be the same. It is interesting to compare imaging at different voltages as well, because it is commonly believed that increasing electron energy will improve the images in thick ice. To compare imaging at different voltages, we fixed the resolution $\left(s_{\mathrm{o}}=4.12 \mathrm{~nm}^{-1}\right)$ and amount of radiation damage. Eq. (5) predicts the voltage dependence of the inelastic scattering, which is known to be proportional to radiation damage [39]. Thereforc, for doing calculations at higher voltages, we increased the clectron incident intensity to keep the number of inelastic (and therefore damaging) events constant. We have ignored the effects of "knock-on" damage at higher voltages, which actually increase the damage.

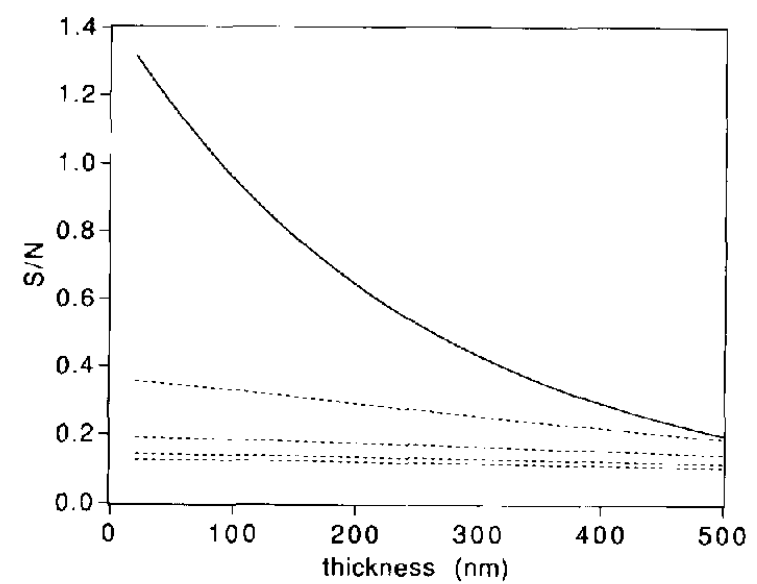

Fig. 6. Calculated signal-to-noise ratio $(\mathrm{S} / \mathrm{N})$ for scattering contrast from $1 \mathrm{~nm}^{2}$ of frozen-hydrated TMV with and without energy filtration: (-) with energy filtration at 80 $\mathrm{keV} ;(. . .-)$ (top to bottom) without filtration at $80,200,400$ and $1000 \mathrm{keV}$. All imaging with $s_{1},=4.12 \mathrm{~nm}^{-1}$ and radiation dose equivalent to 600 electrons $/ \mathrm{mm}^{2}$ at $80 \mathrm{keV}$.

Fig. 6 shows the $\mathrm{S} / \mathrm{N}$ for scattering contrast on TMV imaged with and without energy filtration. At $80 \mathrm{keV}$ with energy filter, a factor of $4 \mathrm{im}$ provement is seen for thin ice, and this improve-


Fig. 7. Cryo-EM images of viruses with and without energy filtration. ( $a, b)$ Low-dose images of frozen-hydrated cottontail papilkoma virus in a $87 \mathrm{~nm}$ layer of ice at $80 \mathrm{keV}, 30300 \times$ magnification, $500 \mathrm{~nm}$ underfocus and 300 electrons $/ \mathrm{nm}^{2}:$ (a) with filtration; (b) without filtration. (c, d) Low-dose images of frozen-hydrated TMV in a $150 \mathrm{~nm}$ layer of ice imaged at $80 \mathrm{keV}$, $30300 \times$ magnification, $1500 \mathrm{~nm}$ underfocus and 600 electrons $/ \mathrm{nm}^{2}$ : (c) with filtration; (d) without filtration. Bar is $50 \mathrm{~nm}$. 
ment decreases as the thickness approaches 500 $\mathrm{nm}$. Over a wide range of thickness, energyfiltered imaging at $80 \mathrm{keV}$ seems to have an advantage, even compared to high-voltage microscopes. For thin ice, the lower-voltage conventional microscopes seem to have an advantage over the high-voltage microscopes. These calculations could be misleading, however, because there are some compensating advantages to high voltage that we have not taken into account, such as: (1) reduced chromatic aberration, (2) reduced effects from specimen charging, (3) reduced multiple scattering and (4) potential for increased coherence of the electrons. In addition, cryo-EM imaging is usually performed with a large amount of objective-lens defocus, which causes a large amount of phase contrast in the image. The amount of phase signal from biological molecules is unchanged by energy filtration, because the number of unscattered and elastically scattered electrons transmitted by the aperture is the same for both microscopes. The amount of phase contrast will always be higher after energy filtration, because of the reduction in the intensity of the background. Phase contrast will be discussed in more detail in section 4 .

\subsection{Practical results of energy filtration upon im- ages of frozen-hydrated molecules}

The calculations of contrast and $\mathrm{S} / \mathrm{N}$ ratios in section 3.1 indicate that energy filtration should noticeably improve the images of frozen-hydrated molecules. This has been shown experimentally by several investigators [37,40-42]. Fig. 7 presents energy-filtered and unfiltered images of cottontail papilloma virus and TMV in ice [41]. The filtered images appear to have less noise. By averaging the image intensities along the axis of a complete TMV particle, we can get a more quantitative view of the signal intensity, background intensity and noise present in images. Fig. 8 shows intensity profiles across a TMV molecule imaged at $\sim 2 \mu \mathrm{m}$ defocus with and without energy filtration. In fig. 8a it is obvious that the ratio of background intensity with and without spectrometcr slit is about 0.5 , showing that half of the electrons in the unfiltered image have lost en-
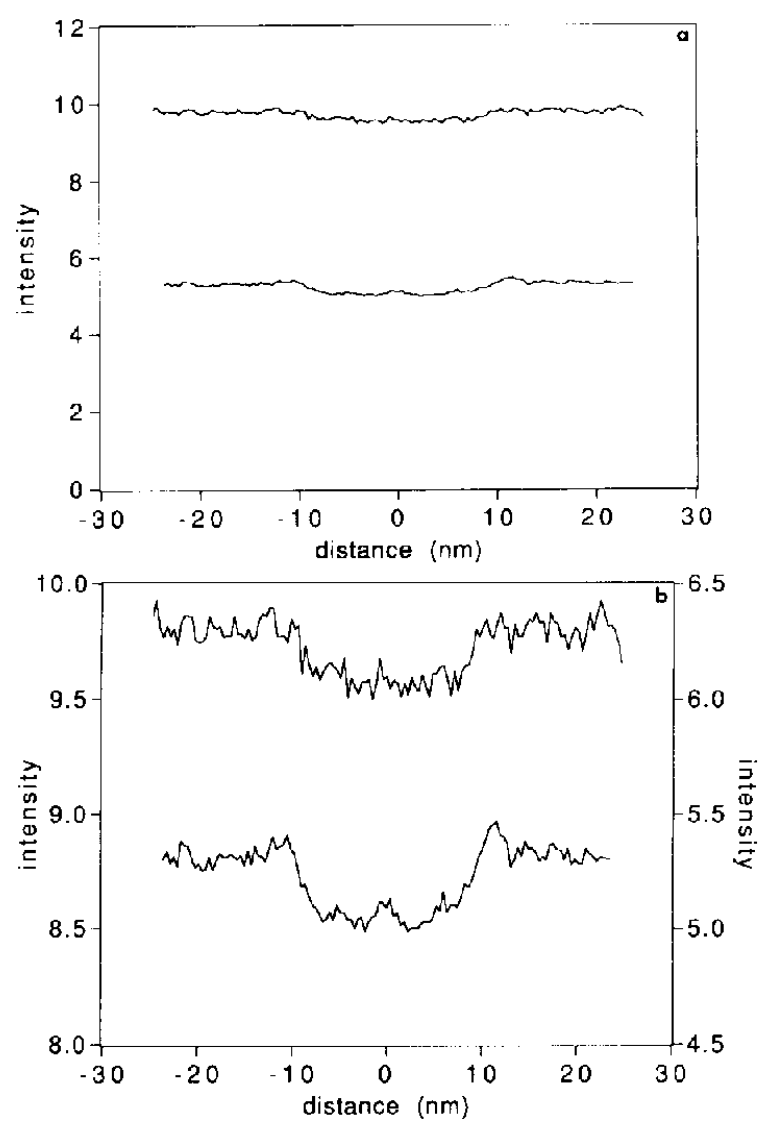

Fig. 8 . Average intensities across the axis of frozen-hydrated TMV in $150 \mathrm{~nm}$ ice at $80 \mathrm{keV}, 30300 \times$ magnification, $\sim 1500$ $\mathrm{nm}$ underfocus and 600 electrons $/ \mathrm{nm}^{2}$. (a) Both intensities on the same scale; (b) expanded, displaced intensity scales. (Top lines) without energy filtration, (bottom lines) with filtration.

ergy. In fig. $8 b$, the intensity scale has been enlarged, so that image detail is visible. The unfiltered image has more noise than the filtered image, even after the statistical noise has been averaged over the $300 \mathrm{~nm}$ length of the molecule. For example, the Fresnel fringe and the hole in the center of the virus is visible only in the filtered image.

\section{Quantitative cryo-EM of TMV}

The discussions in section 2 allow the calculation of the total amount of scattering from a 
biological molecule in ice, but do not describe the distribution of electrons in an image. The positions of the electrons in the image will depend upon microscope defocus, lens aberrations, characteristics of the recording system, etc. In this section we will outline the principles of imaging in the EM, and introduce the simplest approximation for the relationship between molecular densities and image intensities. With this relationship it is possible to predict the image of molecules with known structure or, more importantly, determine the structure of unknown molecules from the image. Using TMV as the example, we will review a recent study of the determination of the mass per unit length and absolute internal densities of biological molecules in ice [37].

\subsection{Image formation}

Image formation in the conventional EM is commonly described in the terms of the first-order theory for scattering contrast and phase contrast $[13,43]$. Scattering contrast arises from the elastically and inelastically scattered electrons removed by the objective aperture. Phase contrast is produced by interference between the unscattercd and elastically scattered electrons transmitted by the objective aperture. However, the theory is only an approximation and it neglects multiple scattering as well as inelastic scattering. Fig. 5 shows that the majority of the electrons in the images of frozen-hydrated samples fall into one or both of these categories. The primary effect of inelastic scattering is to reduce phase contrast by reducing the coherence of the transmitted electrons. A secondary effect is to increase scattering contrast when there is chromatic aberration in the focusing of the energy-loss electrons. One of the important effects of multiple scattering is to increase the effects of chromatic aberration by increasing the scattering angle of the energy loss electrons. Fortunately, energy filtration removes the inelastically scattered electrons, thus eliminating all three effects. Removal of the inelastically scattered electrons reduces the inelastic background from the image (reducing statistical noise) and provides beneficial scattering contrast.
The magnitude of both effects is substantial. The only remaining detrimental effect of inelastic scattcring is a small loss of resolution resulting from the delocalization of the inelastic scattering event $[20,21]$.

\subsubsection{Contrast transfer function theory}

The relationship between object density and phase and scattering contrast is commonly described by the contrast transfer function (CTF), which predicts the loss of information transfer as a function of spatial frequency. The relationship is most easily described in the Fourier transform of the imagc, rather than in the image itself. Under ideal conditions, the primary contributor to the CTF is the electron optics. Contributions can also be made by the delocalization of inelastic scattering, specimen drift, radiation damage, etc. In addition, as ice thickness is increased beyond $150 \mathrm{~nm}$ for the unfiltered bright-field EM or beyond $600 \mathrm{~nm}$ for the energy-filtered bright field EM there are dramatic attenuations of the high spatial frequencies due to beam broadening and chromatic aberration [34]. For the low spatial frequencies of concern in this paper, we will only consider the contributions from the electron optics to the CTF. Two additional factors in recording image intensities must also bc taken into account before quantitation of the images on the computer; the first is due to the scattering of electrons in the photographic emulsion, and the second is due to digitization using finite pixel dimensions. The Fourier transform of the computerized image is the product of the Fourier transform of the object density, the microscope CTF, the film transfer function, and the transfer function of the digitization system. The CTF, which relates the object density to electron intensity, can be estimated by the first-order theory $[13,43]$. The film transfer function, which relates electron intensity to film density, can be estimated [44]. The transfer function of the digitization system, which relates the film density to the digital record, can be modeled by a sinc function appropriate to the pixel dimensions [45]. Because the film and CCD transfer functions are independent of specimen composition and microscope operating conditions, they will be discussed first. 
The microscope CTF, however, depends strongly upon object composition and microscope operating conditions, warranting a more detailed explanation.

\subsubsection{Film transfer function The photo-} graphic record of the image intensities is imperfect. Electron scattering within the emulsion spreads the exposure over many grains. The size and shape of the distribution depend upon the incident electron encrgy and the silver density. The result is attenuation of the higher spatial frequencies, described by a film transfer function. An analytical representation of the film transfer function that fits empirical data quite well has been described [44]:

film transfer function $-F(s)$

$$
=1 /\left[1+\left(s / s_{1 / 2}\right)^{2}\right] \text {, }
$$

where $s$ is the spatial frequency and $s_{1 / 2}$ is the frequency at which the transfer function has a value of one half. When interpreting our film, we have used the values given in ref. [44]. In order to compensate for the film transfer function, we have divided the Fourier transform of the image array by the film transfer function. Using Kodak SO 163 and a magnification of $30300 \times, s_{1 / 2}$ was estimated to be $1.24 \mathrm{~nm}^{-1}$, and therefore the value of $F\left(0.5 \mathrm{~nm}^{-1}\right)$ was 0.86 , which means at the resolution limit of the reconstructions discussed in this paper, the effect of the electron film was small but could not be neglected.

\subsubsection{2. $C C D$ densitometry transfer function}

Densitometry introduces changes in the frequency spectrum of the image, because the imaging device does not sample points but, rather, contiguous areas in the image. The transfer function in this case is the Fourier transform of one sensitive pixel on the CCD. This can be described hy:

$$
\begin{aligned}
& \text { CCD transfer function } \\
& \quad=G\left(s_{x}, s_{y}\right)=\operatorname{sinc}\left(l_{x} s_{x}\right) \operatorname{sinc}\left(l_{y} s_{y}\right),
\end{aligned}
$$

where $l_{x}$ and $l_{y}$ are the pixel dimensions and $s_{x}$ and $s_{y}$ are the spatial frequencies in the $x$ and $y$ directions. In order to compensate for the CCD transfer function, we have divided the Fouricr transform of the image array by the CCD transfer function. Under the conditions of our densitometry $\left(l_{x}=l_{y}=0.4 \mathrm{~nm}\right)$, the value of $G\left(0.5 \mathrm{~nm}^{-1}\right)$ was 0.94 . Thus, at the resolution limit of the reconstructions discussed in this paper, the combined effects of recording film and densitometry were to attenuate the amplitudes by $-20 \%$. Compensation of the digitized image array for these two effects must be done to make the image array a faithful representation of the image intensities.

\subsubsection{Microscope contrast transfer function} According to the first-order theory of the electron microscope CTF $[13,43]$, the relationship between image intensity and object mass density can be expressed in reciprocal space by:

$$
\begin{aligned}
\mathscr{Y}_{\mathrm{i}}(\alpha, \phi)= & -\mathscr{F}_{v}(\alpha, \phi) 2 A(\alpha) f(\alpha) / \lambda\{\sin \chi(\alpha) \\
& \left.+\left[S_{\mathrm{m}}^{\prime} M /(2 A \lambda f(\alpha))\right] \cos \chi(\alpha)\right\},
\end{aligned}
$$

where $\mathscr{F}_{i}(\alpha, \phi)$ is the Fourier transform of the image intensities, $\mathscr{F}_{1}(\alpha, \phi)$ the Fourier transform of the object density, $\phi$ the azimuthal angle, $A(\alpha)$ the objective aperture function ( 1 for $\alpha<\alpha_{0}$, 0 for $\left.\alpha>\alpha_{0}\right), f(\alpha)$ the molecular scattering amplitude, $A$ is Avogadro's number and

$\chi(\alpha)=2 \pi / \lambda\left(C_{\mathrm{s}} \alpha^{4} / 4+\Delta f \alpha^{2} / 2\right)$,

where $C_{\mathrm{s}}$ is the coefficient of spherical aberration and $\Delta f$ the defocus.

The term in brackets is referred to as the microscope contrast transfer function,

$\mathrm{CTF}=[\sin \chi(\alpha)+Q(\alpha) \cos \chi(\alpha)]$,

where

$Q(\alpha)=\left[S_{\mathrm{m}} M / 2 A \lambda f(\alpha)\right]$.

The function $\sin \chi(\alpha)$ is commonly referred to as the phase contrast transfer function and $Q(\alpha) \cos \chi(\alpha)$ as the seattering contrast transfer function. Both are commonly expressed as a function of spatial frequency. Usually $f(\alpha)$ and $Q(\alpha)$ are considered constant over the frequency range of relevance to biological applications, and the 
effects of spatial and temporal coherence are ignored, because they are expected to be negligible at low resolution [46]. $Q$ represents the maxi-
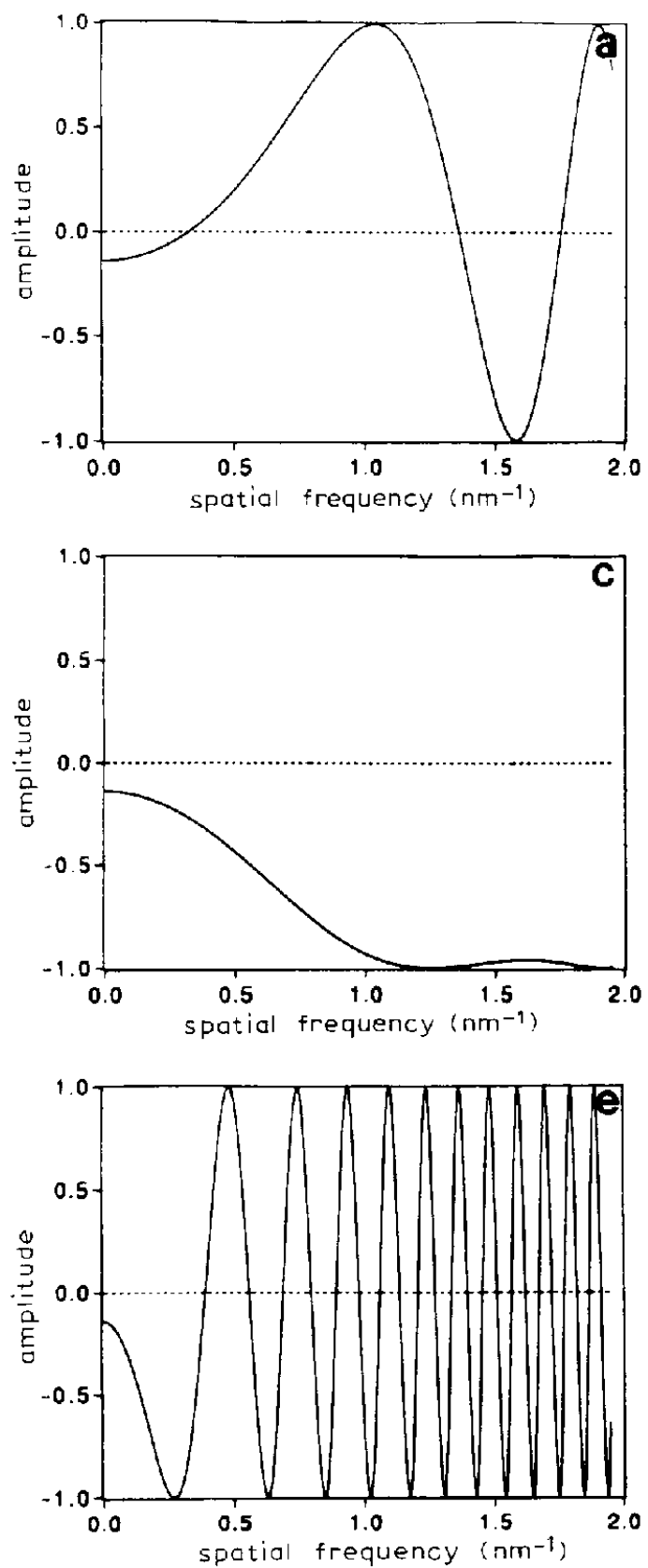

mum contribution from scattering contrast relative to that from phase contrast. Because $S_{\mathrm{m}}$ is increased by energy filtration (see table 3 ), $Q$ will
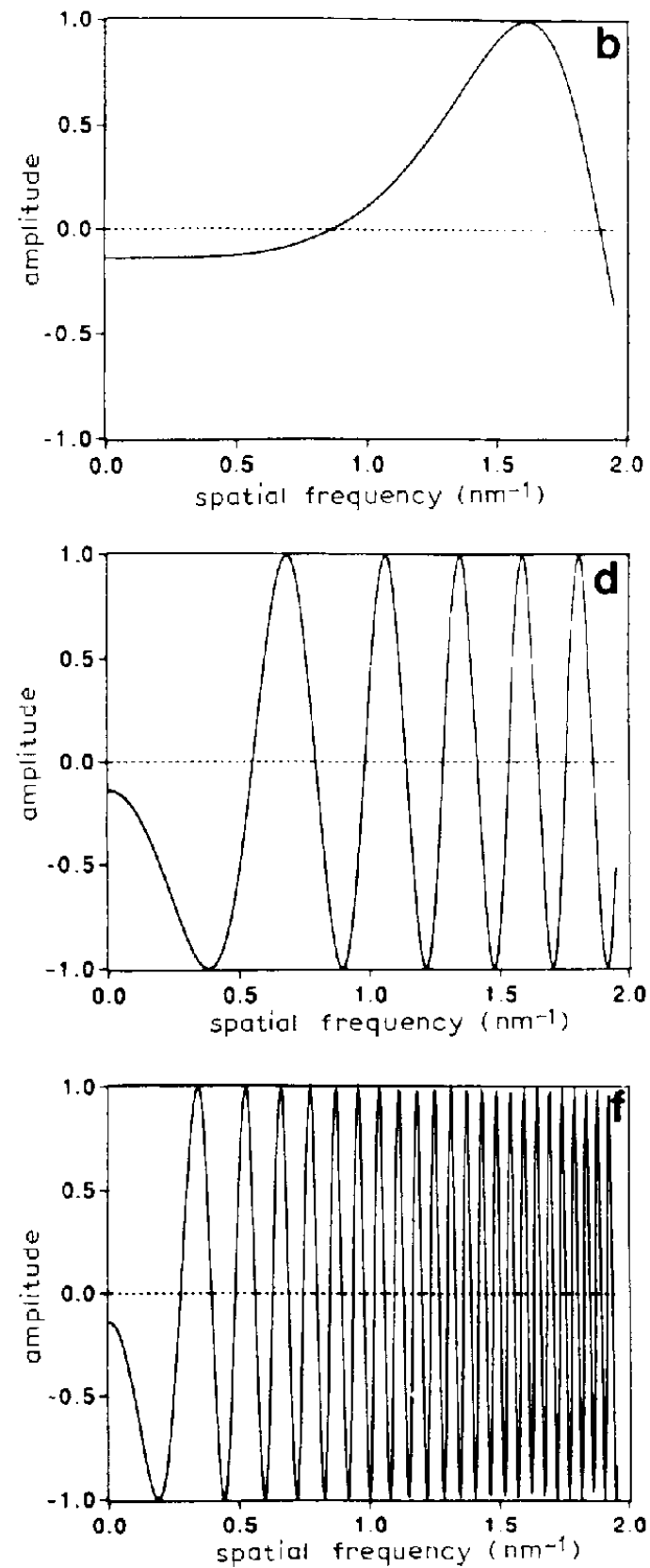

Fig. 9. Calculated CTF at different defoci. The values of eq. (32) are plotted assuming the instrumental parameters of the Zeiss $\operatorname{EM} 902\left(\lambda=0.0042 \mathrm{~nm}, C_{\mathrm{s}}=2.2 \mathrm{~mm}\right)$, (a) $\Delta f=-100 \mathrm{~nm}$, (b) $\Delta f=0 \mathrm{~nm}$, (c) $\Delta f=\Delta f_{\mathrm{opt}}=100 \mathrm{~nm}$, (d) $\Delta f=750 \mathrm{~nm},(\mathrm{e}) \Delta f=1500$ $\mathrm{nm}$ and (f) $\Delta f=3000 \mathrm{~nm}$. 

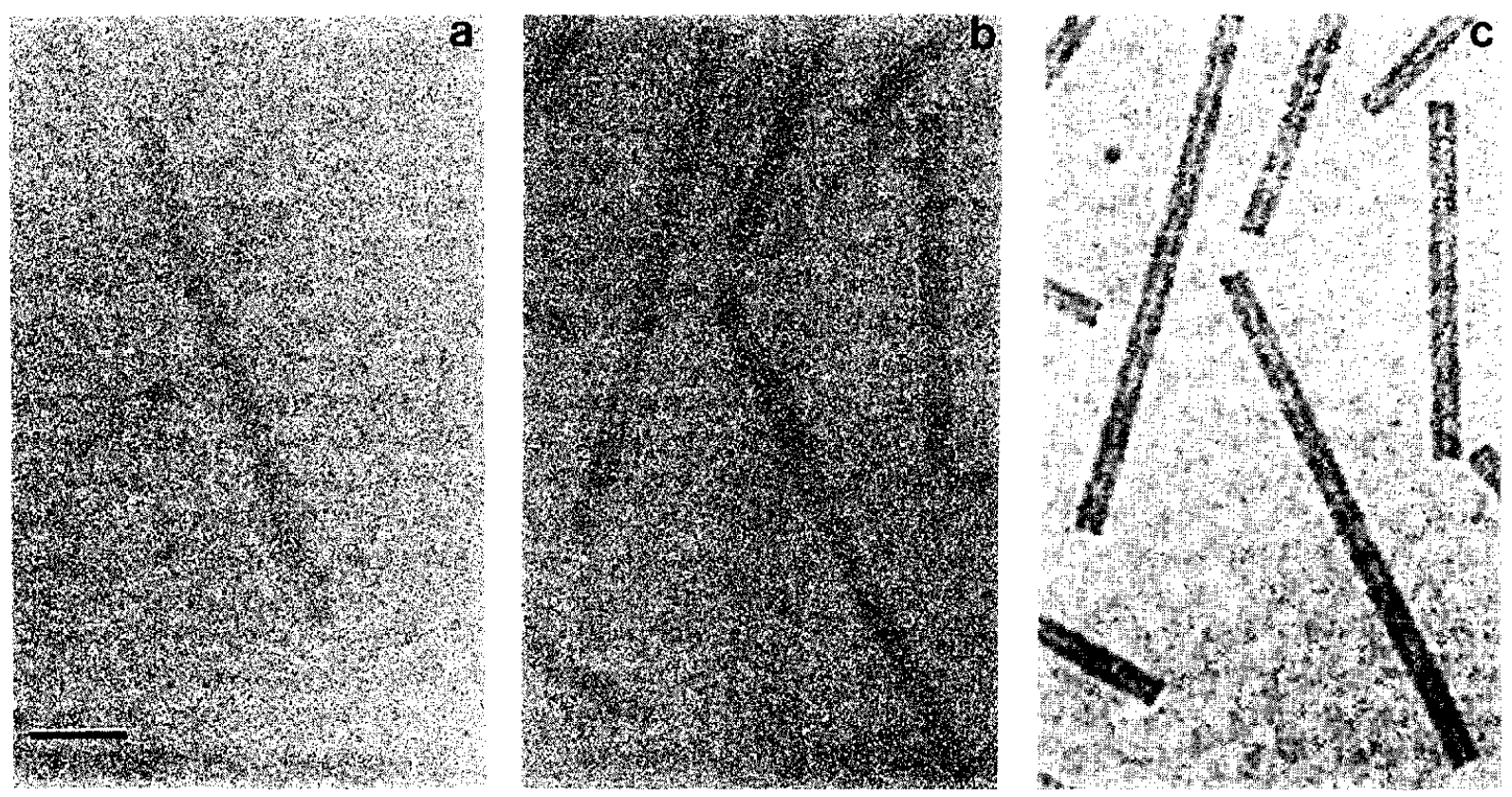

Fig. 10. Images of frozen-hydrated TMV at different values of defocus. Original magnification $30.300 \times:$ dose $=600 \mathrm{electrons} / \mathrm{nm}^{2}$. (a) $200 \mathrm{~nm}$ defocus, (b) $780 \mathrm{~nm}$ defocus, and (c) $4620 \mathrm{~nm}$ defocus. Bar is $50 \mathrm{~nm}$.
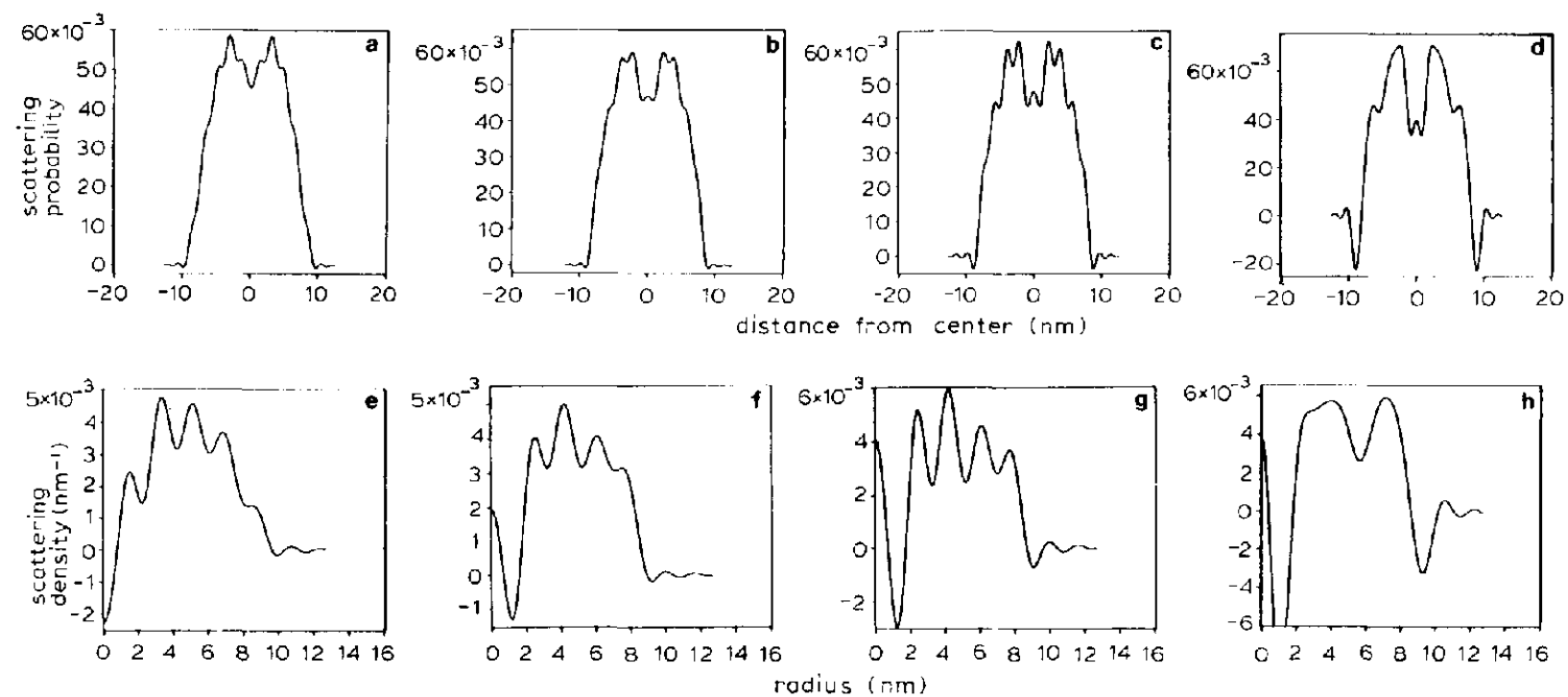

Fig. 11. Predicted $1.9 \mathrm{~nm}$ resolution projections and reconstructions of frozen-hydrated TMV at different values of defocus. (a-d) Projected scattering probabilities at defocus values of (a) $-100 \mathrm{~nm},(\mathrm{~b}) 30 \mathrm{~nm}$, (c) $100 \mathrm{~nm}\left(\Delta f_{\text {opt }}\right.$ ) and (d) $780 \mathrm{~nm}$. (e-h) Radial scattering densities calculated from the projections in $(\mathrm{a}-\mathrm{d}) .1 .9 \mathrm{~nm}$ resolution. From ref. [37]. 
also be increased. The value of $Q$ might also depend upon ice thickness. We will ignore the fact that the first-order theory is not rigorously correct, and treat eq. (30) as a useful phenomenological description of the images. This amounts to combining all contributions from the imaginary part of the complex scattering amplitudes, removal of some of the scattered electrons by the objective aperture, and removal of the inelastically scattered electrons by the spectrometer into one quantity loosely called scattering contrast. To use the equation for image compensation we will cmpirically determine a value of $Q$ that best describes changes in the images due to defocus of the objective lens.

Fig. 9 shows the calculated CTF for several values of defocus, using an empirical value of $Q=0.14$ (see section 4.1.2). It is evident that certain spatial frequencies will be attenuated or reversed in contrast. Perfect transfer of information would correspond to a CTF with a value of unity for all spatial frequencies. The defocus that results in a CTF with a value of unity throughout the largest range of spatial frequency is $\Delta f_{\text {opt }}=$ $\left(C_{\mathrm{s}} \lambda\right)^{1 / 2}$ [47]. However, as shown in fig. 9, even at this defocus there is serious attenuation of the low spatial frequencies. Thus, compensation for the CTF is required.

\subsubsection{Contrast transfer function practice}

$Q$ can be experimentally determined from two images of the same biological molecules taken with different values of defocus. This method yielded a value of $Q=0.14$ for energy-filtered images of TMV in $150 \mathrm{~nm}$ of icc [37]. A value of $Q=0.07$ was determined (without energy filtration) by Toyoshima and Unwin for ice thicknesses of $30-50 \mathrm{~nm}$ [14].

The effects of the CTF on images of frozen-hydrated TMV are shown in fig. 10. The low signalto-noise ratio for images at or near focus is evident in fig. 10a which shows frozen-hydrated TMV at $200 \mathrm{~nm}$ defocus. At $780 \mathrm{~nm}$ defocus (fig. 10b) the signal is significantly increased, the the CTF is optimized for the $2.3 \mathrm{~nm}$ repeat of the TMV. At $4620 \mathrm{~nm}$ defocus (fig. 10c) the $2.3 \mathrm{~nm}$ repeat is no longer present, while the central hole and Fresnel fringes show up clearly. Fig. 11 shows calculated projections from model TMV images at defocus values of $-100,30,100$ and $780 \mathrm{~nm}$. Modeling of the TMV images is discussed in detail in ref. [37], which tested the validity of the model by comparing the predicted and observed $\mathrm{X}$-ray scattering from TMV. It is evident that minimal overfocus or underfocus substantially modifies the TMV images. The effects are much more dramatic at the defocus values of $2-4 \mu \mathrm{m}$ commonly used for cryo-EM.

\subsection{Image compensation}

The CTF has the effect of altering the image intensity at different spatial frequencies. For those spatial frequencies where appreciable information is transferred, the intensity distortions can be compensated by dividing the Fourier transform of the images by the calculated CTF. The validity of the first-order theory of Erickson and Klug [13] for predicting and compensating the effects of the CTF has recently been demonstrated by comparing the equatorial Fourier transforms from calculated and empirical images of frozen-hydrated TMV [37]. Fig. 12a compares the Fourier transform of the model for TMV scattering (dashed line) with that of an observed image of TMV in $150 \mathrm{~nm}$ of ice at $780 \mathrm{~nm}$ defocus (solid line). Fig. 12b compares the transform of the model (dashed line) with the observed image after compensation of the CTF (solid line). The large differences between the relative peak heights in fig. 12a illustrate the necessity for compensation. After compensation, the agreement between the model and experimental Fourier transform is excellent out to the resolution limit of $1.9 \mathrm{~nm}$, despite the fact that there is appreciable multiple scattering and contrast from inelastic scattering. The crystallographic $R$ factor between the Fourier transforms of the cryo-EM images and the model of TMV was 0.12 for $0 \leqslant s \leqslant 0.5 \mathrm{~nm}^{-1}[37]$.

The fidelity of the compensated images is further demonstrated in fig. 13, which compares the projected scattering probabilities of TMV at 780 $\mathrm{nm}$ defocus (fig. 13a), the same experimental probabilities after compensation (fig. 13b, solid line), and the predicted probabilities at the same 
resolution (fig. 13b, dashed line). There is little resemblance between the projection obtained from the defocused image data and that predicted theoretically. The projection obtained after CTF compensation, however, closely resembles the theoretical projection. The excellent agreement between the calculated image and the observed image after CTF compensation reflects the accuracy of the model and scattering calculations, and the adequacy of the first-order theory to compensate the images from very low spatial frequencies to the resolution limit of $1.9 \mathrm{~nm}$ [37].

\subsection{Determination of absolute mass}

Cryo-EM has the potential to accurately determine the absolute mass of biological molecules without the use of an internal standard [37]. Absolute scattering probabilities can be related directly to mass per unit area using the calculated values of the mass scattering coefficient, $S_{\mathrm{m}}$ (see sections 2.2.1. and 2.2.2). Summation of the mass per unit area over the molecular area yields the total molecular mass, from which the mass per unit length can be derived. Table 3 is a summary of the theoretical mass scattering coefficients of ice and biological material in vacuum and in vitreous ice, containing averages over all the atoms of each type of molecule.

Fig. 13b shows observed and predicted absolute scattering probabilities of frozen-hydrated TMV [37]. The integrated scattering probability per unit length was 0.75 and $0.74 \mathrm{~nm}$ for the cryo-EM data and model, respectively. Thus, the observed molecular scattering cross section agreed quantitatively with that predicted. The theoretical mass scattering coefficients in table 3 also predict the correct mass per unit length. Thus energy-filtered cryo-EM should be an effective tool for determining the absolute mass and mass densities of biological molecules, without the inherent limitations to resolution that affect use of dark-field STEM.

\subsection{Determination of radial densities}

If the calculations of mass scattering coefficients and compensation for the transfer func-
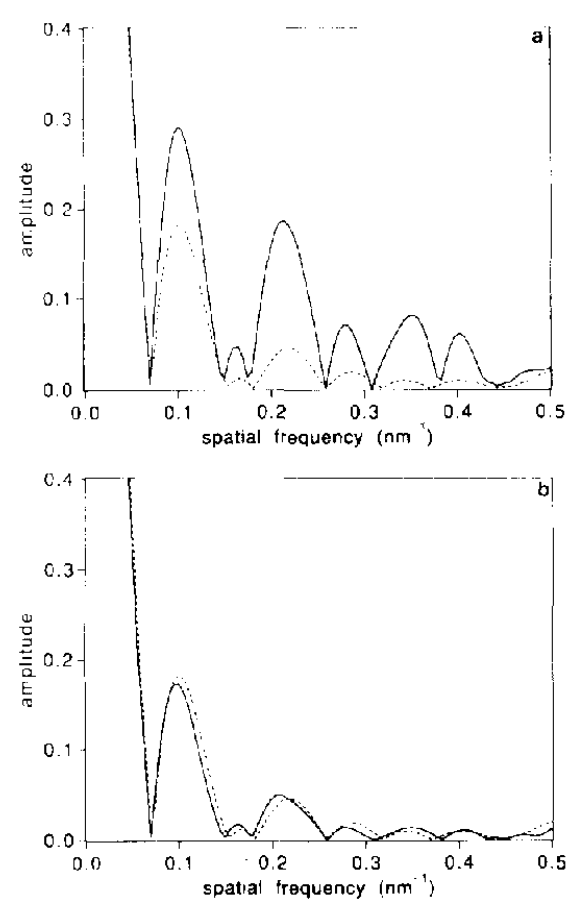

Fig. 12. Observed and predicted one-dimensional Fourier equatorial transforms of frozen-hydrated TMV. (a) Fourier transform of the observed image with $780 \mathrm{~nm}$ defocus (- ); Fourier transform of the predicted scattering from TMV (-....). (b) Fourier transform of the observed image after CIT compensation (-__- ) ; Fourier transform of the predicted scattering from IMV (-..-). Comparison of the observed and predicted Fourier amplitudes gave a crystallographic $R$ factor of 0.12 , where $R=\sum \mid F_{\text {obs }}-F_{\text {pred }}^{\prime} / \sum F_{\text {olls. }}$. CTFs were calculated assuming $Q=0.14$. Fourier transforms were normalized to unity at zero spatial frequency. $1.9 \mathrm{~nm}$ resolution. Data from ref. [37].

tions of the electron microscope, photographic film, and densitometry are accurate, it should be possible to reconstruct the internal mass densities of biological molecules from the images. The most stringent test of the calculations and procedures is to reconstruct the internal densities of a known structure, such as TMV. This can be done most cffectively over a wide range of spatial frequencies by performing a radial density reconstruction from the defocused images, because of the continuity of information along the equator of the Fourier transform of the molecule (fig. 12). If cryo-EM is able to correctly determine densities 

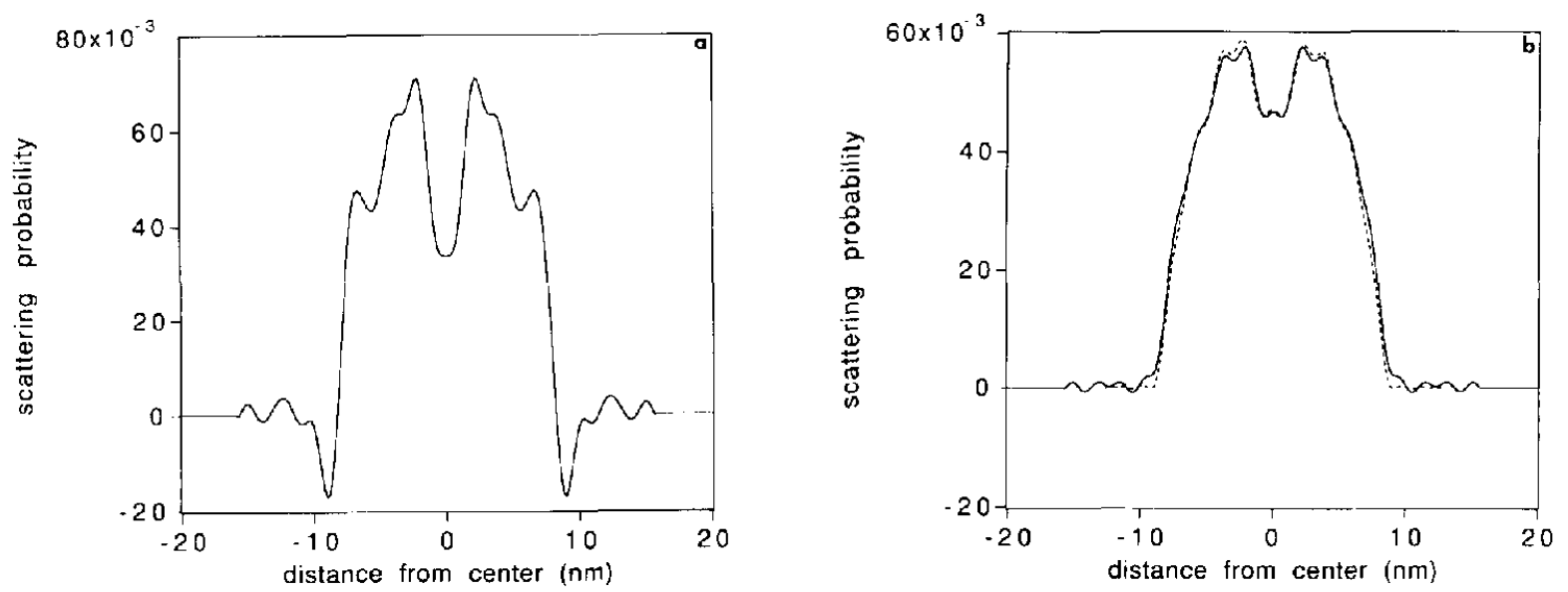

Fig. 13. Effects of CTF compensation on projections of frozen-hydrated TMV. (a) Observed scattering probabilities of TMV at 780 $\mathrm{nm}$ defocus. (b) Comparison of the observed scattering probabilities after CTF compensation ( $\longrightarrow$ ), and the predicted scattering probabilities (-...-). $1.9 \mathrm{~nm}$ resolution. From ref. [37].

in one dimension, it should have the same abilities in the other two dimensions as well.

Radial reconstructions of TMV were calculated from the projected scattering probabilities using a real-space algorithm [37]. The radial reconstructions represents scattering densities, which should, in the case of the energy-filtered images, be nearly proportional to mass densities (table 3). The scattering densities depend strongly

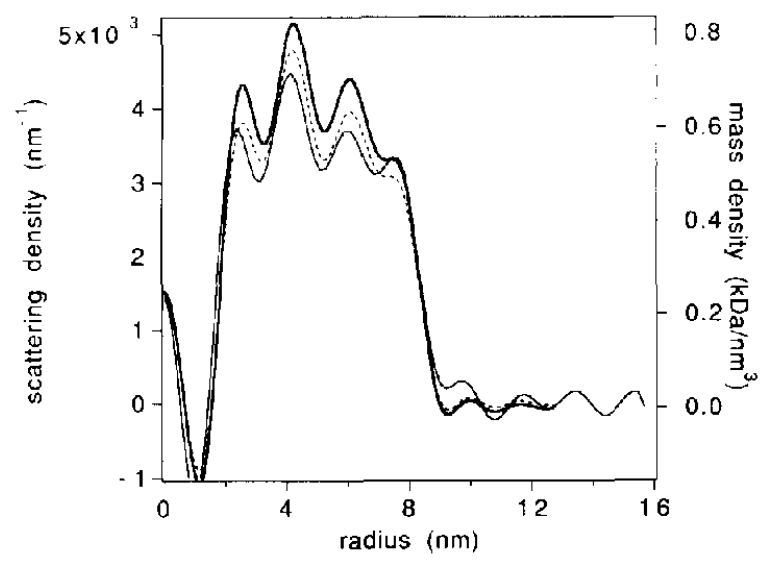

Fig. 14. Comparison of predicted mass densities with experimental and predicted scattering densities of TMV at $1.9 \mathrm{~nm}$ resolution. (— $)$ Predicted radial mass densities; (-.....) predicted radial scattering densities; (-) CTF-compensated experimental radial scattering densities. From ref. [37]. upon solvent composition and density. In addition, the relationship between scattering and mass is slightly different for phase and scattering contrast. In theory, the ratio of scattcring to phase contrast is larger for atoms of higher atomic number, and larger for thicker layers of ice.

Fig. 14 compares the predicted radial mass densities, predicted radial scattering densities and the experimental radial scattering densities of TMV. The predictions have been calculated from first-order theory [13] using the X-ray coordinates [16]. The peaks at $2.5,4.2,6.0$ and $7.5 \mathrm{~nm}$ radius can be attributed to the a series of reverse turns, the RNA, the LS, RS, LL and LR helices, and the $\mathrm{C}$ helix [16]. The scattering densities from the compensated cryo-EM data appear to be a valid representation of the radial mass-density distribution of both RNA and protein. The relative heights of the RNA and protcin peaks would have been significantly different if the TMV had been surrounded by vacuum or glucose, as shown in fig. 15. Thus, ice is an ideal medium for quantitative electron microscopy of mixtures of nucleic acids and proteins. The accuracy of the reconstruction of the internal density distribution of TMV shows that quantitative EM of frozen-hydrated molecules is a reality.

How do the cryo-EM radial reconstructions of TMV compare with results by STEM? Fig. 16 
compares the cryo-EM results with earlier STEM results [48]. The STEM reconstruction compares poorly with the cryo-EM results and with the predictions of radial density distribution (fig. 14). Better STEM reconstructions were obtained by Steven et al., but only by selection of those few images that had a definite central hole [49]. Such selection of images could not be justified for molecules of unknown structure. However, even the STEM reconstructions of selected images (e.g., fig. 6 of ref. [49]) do not accurately portray the peak positions and peak densities of TMV, presumably due to the effects of dehydration on even the best preserved virus particles.

\section{Current issues for quantitative electron mi- croscopy of frozen-hydrated molecules}

In this paper we have shown the relationships between specimen mass density and electron scattering probabilities and how they can be utilized to determine the absolute densities and masses of biological molecules. Simple equations were presented in order to facilitate a quantitative understanding of image contrast in vacuum and in ice. A method for calculating the intensities for thick specimens was discussed, and applied successfully to understanding the image intensitics of thick layers of ice. These results were
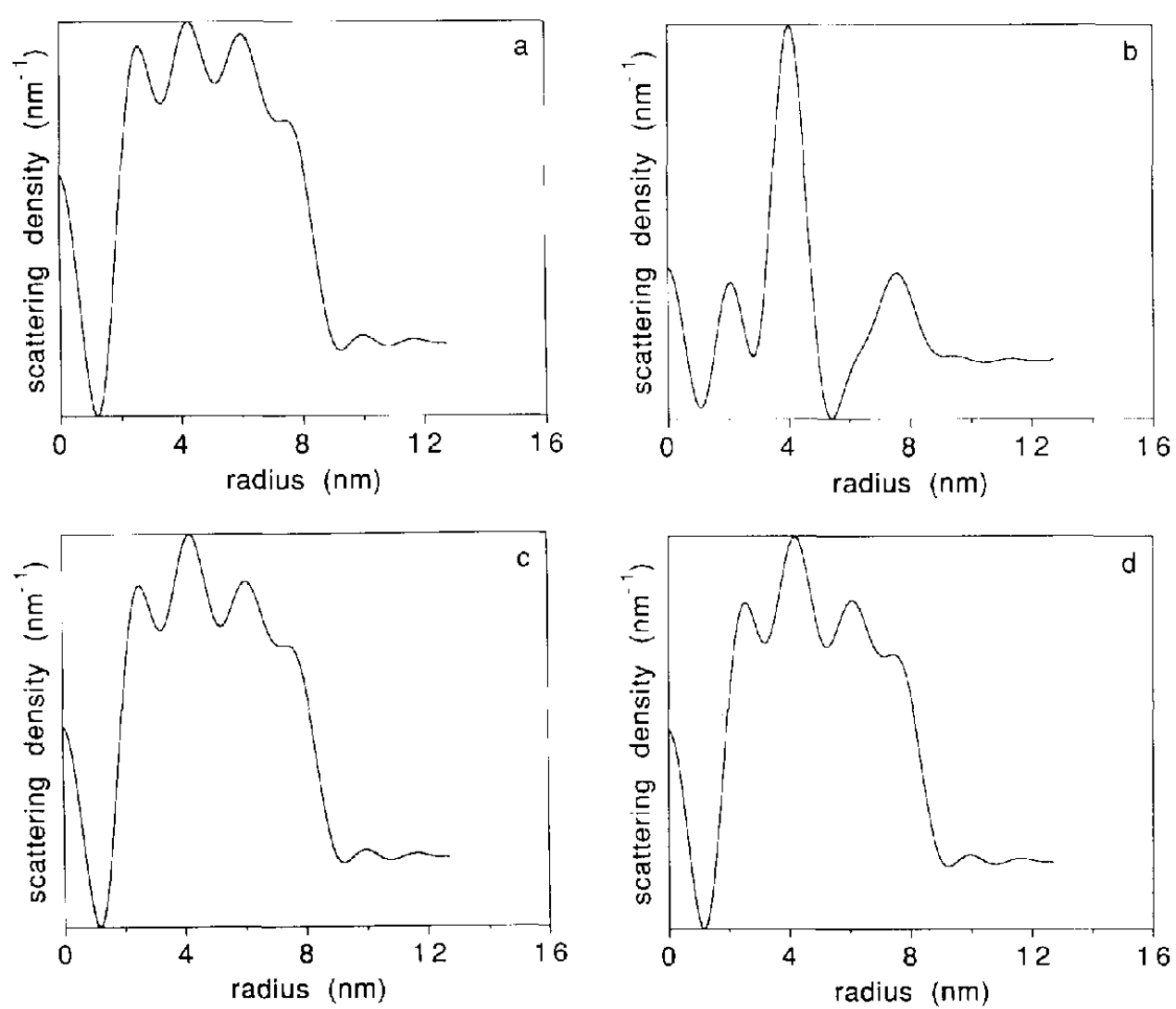

Fig. 15. Predicted radial scattering densities of TMV in different media; (a) vacuum; (b) glucose; (c) water with density $0.82 \mathrm{~g} / \mathrm{cm}^{3}$; (d) water with density $1.02 \mathrm{~g} / \mathrm{cm}^{3}$. Models arbitrarily scaled. $1.9 \mathrm{~nm}$ resolution. 


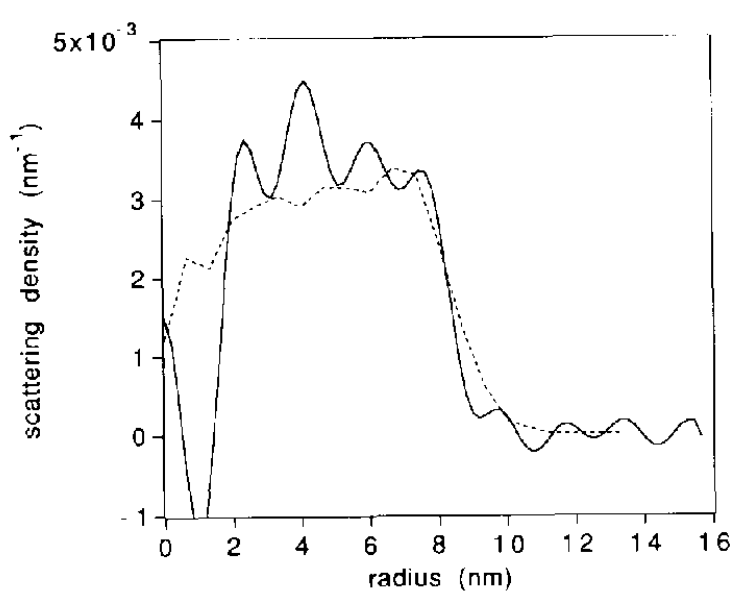

Fig. 16. Observed radial reconstructions of TMV hy cryo-EM and STEM. (—— CTF-compensated radial scattering densities of frozen-hydrated TMV imaged by cryo-EM [37]. (-....) Radial scattering densities of frozen-dried TMV imaged by STEM [49].

used to predict the advantages of energy filtration on electron imaging. Quantitative analyses of TMV images showed that a simple compensation for the contrast transfer function is necessary and sufficient to accurately reconstruct the internal densities of biological molecules to at least $2 \mathrm{~nm}$ resolution. Thus there should no longer be any doubt that accurate reconstructions can be made at low resolution.

The empirical ratio of scattering to phase contrast is about 0.14 in the energy-filtered images of biological molecules in ice [37]. However, the table values of the atomic scattering amplitudes predict a ratio of only 0.085 . It is clear from our absolute scattering measurements that this discrepancy is not due to an error in the estimation of the mass scattering coefficient. The ability to correctly compensate the images secms to validate the form of eq. (30). Therefore, the value for the scattering amplitude in the forward direction might not be correct. The single atom amplitudes might be in error due to the failure to represent the electronic structure of the solid specimens. Measurements of scattering amplitude at very small angles are difficult to perform due to inelastic scattering. The energy-filtered EM should be able to resolve this issue.
STEM has been able to localize heavy-atom labels in many situations [50-52], and there are now qualitative results from cryo-EM as well [53]. It would be desirable to compensate for the $\mathrm{CTF}$ in images of molecules containing heavy atoms, but fundamental obstacles remain. The calculated values for $Q$ depend strongly on atomic number. If this is true, it becomes difficult to compensate for the CTF of molecules of unknown structure, which might contain heavy atoms. If the compensation were to be done using a value of $Q$ that was appropriate for the organic part of the molecule, the heavy atoms would be greatly underrepresented in the image reconstructions. Calculations and experiments on heavy-atom labeled molccules of known structure are necessary to resolve this issue.

Finally, there is the issue of compensation for the CTF at higher spatial frequencies. For biological crystals as well as for TMV, there are very severe differences between the high-spatialfrequency amplitudes observed from $\mathrm{X}$-ray electron diffraction and those found in Fourier transforms of electron images [54,55]. The amplitudes determined from the images decrease rapidly at higher spatial frequencies. This effect has been attributed to beam-induced specimen and image motion, both perhaps the result of specimen charging. In the case of our TMV data, the information was limited to less than $1.9 \mathrm{~nm}$, because of the inherently low amplitudes of the equatorial Bessel functions at higher spatial frequencies. In order to get an idea of the contrast transfer at higher spatial frequencies, it is necessary to compare the near-meridional amplitude on the layer lines of the TMV Fourier transforms. A convenient measure of comparison is $R$, the ratio of the maximum amplitudes on the 6 th and 3 rd layer lines [55]. Our TMV model predicts that $R=1.06$ for the energy-filtered cryo-EM images and $R=1.41$ for the unfiltered images [37]. In fact, we are barely able to identify the oth layer line in our energy-filtered data and our experimental value for $R$ is $\sim 0.03$. Part of this unexpected attenuation at higher spatial frequencies might be the result of delocalization of inelastic scattering. Spatial resolution between 0.3 and 0.5 $\mathrm{nm}$ has been measured for energy losses of about 
$100 \mathrm{eV}[56,57]$, but the resolution including lower losses has been measured to be only 1-2 nm [20], which seems to agrec with simple theory [21]. The extent to which this effect attenuates the higher spatial frequencies of energy-filtered (as well as unfiltered) images has not yet been tested, although dark-field images with electrons having lost various amounts of energy would determine the limitations to the use of inelastic scattering.

\section{Acknowledgements}

We thank Dr. T. Baker, Mr. N. Olson and Ms. D. Rankert for the cottontail papilloma virus images, and Ms. K. Nolta for preliminary analysis of the images of vitreous ice. We also thank Dr. R. Henderson for very useful discussions and Carl Zeiss Inc. for technical advice and assistance. These studies were supported by NIH GM27937, NSF DIR8706052 and NSF DMB 9106659 .

\section{References}

[1] R. Henderson. J. Baldwin and T. Ceska, J. Mol. Biol. 213 (1990) 899.

[2] F. Zcitler and G.F. Bahr, Fxp. Cell Res. 12 (1957) 44.

[3] E. Zeitler and G.F. Bahr, J. Appl. Phys. 33 (1962) 847 .

[4] G. Brakenhoff, Relative mass determination in darkfield microscopy, in: Principles and Techniques of Electron Microscopy Bislogical Applications, Ed. M. Hayat (VanNostrand/Reinhold, New York, 1974) p. 16.

[5] M.K. Lamvik and J.P. Langmore, in: Scanning Electron Microscopy/1977. Vol. 1, Ed. O. Johari (IITRI, Chicago. 1977) ก. 401.

[6] J. Wall and J. Hainfeld, Annu. Rev. Biophys. Biophys. Chem. 15 (1986) 355 .

[7] K. Taylor and R. Glaeser, Science $106(1974) 1036$.

[8] K. Taylor and R. Glacser, J. Ultrastruct. Res. 55 (1976) 448.

[9] J. Dubochet, J. Lepault, R. Freeman, J. Berriman and J. Homo, J. Microscopy 128 (1982) 219.

[10] W. Chiu. Annual Rev. Biophys. Biophys. Chem. 15 (1986) 237.

[11] M. Stcwart and G. Vigers, Nature 319 (1986) 631.

[12] J. Dubochet, M. Adrian, J. Chang. J. Ilomo, J. I.cpault, A. McDowall and P. Schultz, Q. Rev. Biophys. 21 (1988) 129.

[13] H. Erickson and A. Klug, Phil. Trans. Roy. Soc, (London) B $261(1971) 105$.
[14] C. Toyoshima and N. Unwin. Ultramicroscopy 25 (1988) 279 .

[15] (C. Toyoshima and N. Unwin, J. Cell Biol. I11 (1990) 2623.

[16] K. Namba, R. Pattanayek and G. Stubbs, J. Mol. Biol. 208 (1989) 307.

[17] J. I.epault, J. Microscopy 140 (1985) 73.

[18] 'T.-W. Jeng, R. Crowther, G. Stubbs and W. Chiu, J. Mol. Biol. 205 (1989) 251.

[19] L. Reimer and M. Ross-Messemer, J. Microscony 155 (1989) 169 .

[20] M. Isaacson, J.P. Langmore and H. Rose. Optik 41 (1974) 92.

[21] H. Kohl and 11. Rose. Adv. Electron. Flectron Phys. 65 (1985) 175

[22] L. Schater. A. Yates and R. Bonham, J. Chem. Phys. 55 (1971) 3055

[23] J.P. Langmore, J.S. Wall and M.S. Isaacson. Optik 38 (1973) 335

[24] J. Wall. M. Isaacson and J. Langmore. Optik .39 (1474) 359.

[25] R. Castaing, Energy filtering in electron microscopy and electron diffraction, in: Physical Aspects of Electron Microscopy and Microbeam Analysis, Ed. B. Siegel (Wiley, New York, 1975$)$ p. 287.

[26] I: Ottensmeyer and J. Andrew, J. Ultrastruct. Res. 72 (1980) 336 .

[27] F. Otlensmeyer, J. Ultrastruct. Res. 88 (1984) 121.

[28] I.. Reimer. Ultramicroscopy 31 (1989) 169.

[29] F.P. Ottensmeyer, Ann. NY Acad. Sci. 483 (1992) 339.

[30] J. Badger and D.L.D. Caspar, Proc. Natl. Acad. Sci. 88 (1991) 622 .

[31] M.G. Sceats, S.A. Rice, Water, Vol. 7 (Plenum, Vew York, 1982).

[32] A. Hallbrucker, E. Mayer, L.P. O'Mard. J.C. Dore and P. Chieux. Phys. Letl. A $159(1991) 406$.

[33] A.V. Crewe and T. Groves, J. Appl. Phys. 45 (1974) 3662

[34] T. Groves, Ultramicroscopy 1 (1975) 15

[35] L. Reimer and P. Gentsch, Cltramicroscopy 1 (1975) 1.

[36] B.D. Athey. M.F. Smith, D.A. Rankert, S.P. Williarms and J.P. Langmore, I. Cell Biol. 111 (1990) 795.

[37] M.F. Smith and J.P. Langmore, J. Mol. Biol. 226 (1492), in press.

[38] R.T. Simpson, Nature $343(1990) 387$.

[39] M. Isaacson. Specimen damage in the electron microscope, in: Principles and Techniques of Electron Microscopy, Ed. M.A. Hayat (Van Nostrand/Reinhold, New York, 1975$)$ p. 1.

[40] J. Lamgmore and B.I). Athey, in: Proc. 45th Annual EMSA Meeting, Baltimore. MD, 1987, Ed. G.W. Bailey (San Franciseo Press, San Francisco, 1987) p. 252.

[41] J. I angmore, M.F. Smith and D.A. Rankert, in: Prec. 12th Int. Congr. for Llectron Microscopy, Lds. L. Peachey and D. Williams (San Francisco Press, San lrancisco, 1990) p. 652 .

[42] R. Schroder, W. Hofmann and J.-F. Ménétret, J. Struct. Bicl. $105(1990) 28$. 
[43] II. Erickson, The Fourier transform of an electron micrograph - first order and second order theory of image formation, in: Advances in Optical and Electron Microscopy (Academic Press, New York, 1971) p. 163.

[44] K. Downing and D. Grano, Ultramicroscopy 7 (1982) 381.

[45] E.O. Brigham, The Fast Fourier Transform (Prentice Hall, New Jersey, 1973).

[46] J. Frank, Optik 38 (1973) 519

[47] O. Scherzer, J. Appl. Phys. 20 (1949) 20.

[48] M.F. Smith, B.D. Athey, S.P. Williams and J.P. Langmore, J. Cell Biol. $110(1990) 245$.

[49] A. Steven, J. Hainfeld, B. Trus, P. Steinert and J. Wall, Proc. Natl. Acad. Sci. 81 (1984) 6363.

[50] J.S. Wall, P.A. Bartlett and S.J. Singer, Ultramicroscopy $8(1982) 397$
[51] K. Monson, J. Wall and J. Hainfeld, Ultramicroscopy 21 (1987) 147

[52] J. Hainfeld, Science 236 (1987) 450.

[53] R. Milligan, M. Whitlaker and D. Safer, Nature 348 (1990) 217.

[54] R. Henderson and R. Glaeser, Ultramicroscopy 16 (1985) 139.

[55] R. Henderson, Ultramicroscopy 46 (1992) 1.

[56] K. Adamson-Sharpe and F. Ottensmeyer, J. Microscopy 122 (1981) 309

[57] M.S. Isaacson and M. Scheinfein, in: Proc. 43rd Annual EMSA Meeting, I ouisville, KY, 1985, Fd. G.W. Bailey (San Francisco Press, San Francisco, 1985) p. 396. 